Draft Version September 7, 2018

Preprint typeset using $\mathrm{IATEX}_{\mathrm{E}} \mathrm{X}$ style emulateapj v. 12/14/05

\title{
A DEEP, WIDE-FIELD, AND PANCHROMATIC VIEW OF 47 TUC AND THE SMC WITH HST: OBSERVATIONS AND DATA ANALYSIS METHODS ${ }^{1}$
}

Jason S. Kalirai ${ }^{2,3}$, Harvey B. Richer ${ }^{4}$, Jay Anderson ${ }^{2}$, Aaron Dotter ${ }^{2}$, Gregory G. Fahlman ${ }^{5}$, Brad M. S. Hansen ${ }^{6}$, Jarrod Hurley ${ }^{7}$, Ivan R. King ${ }^{8}$, David Reitzel ${ }^{6}$, R. Michael Rich ${ }^{6}$, Michael M. Shara ${ }^{9}$, Peter B. Stetson ${ }^{5}$, And Kristin A. Woodley ${ }^{4}$

Draft version September 7, 2018

\begin{abstract}
In Hubble Space Telescope (HST) Cycle 17, we imaged the well known globular star cluster 47 Tucanae for 121 orbits using the Wide Field Channel (WFC) of the Advanced Camera for Surveys (ACS) and both the UVIS and IR channels of the newly installed Wide Field Camera 3 (WFC3) instrument (GO-11677, PI - H. Richer). This unique data set was obtained to address many scientific questions that demand a very deep, panchromatic, and panoramic view of the cluster's stellar populations. In total, the program obtained over $0.75 \mathrm{Ms}$ of imaging exposure time with the three $H S T$ cameras, over a time span of 9 months in 2010. The primary ACS field was imaged in the two broadband filters $F 606 \mathrm{~W}$ and $F 814 \mathrm{~W}$ filters, at 13 orientations, for all 121 orbits. The parallel WFC3 imaging provides a panchromatic $(0.4-1.7$ micron) and contiguous imaging swath over a 250 degree azimuthal range at impact radii of $6.5-17.9 \mathrm{pc}$ in 47 Tuc. This imaging totals over $60 \mathrm{arcmin}^{2}$ in area and utilizes the $F 390 W$ and $F 606 W$ broadband filters on WFC3/UVIS and the $F 110 W$ and $F 160 W$ broadband filters on WFC3/IR.
\end{abstract}

In this paper, we describe the observational design of the new survey and one of the methods used to analyze all of the imaging data. This analysis combines over 700 full-frame images taken with the three HST cameras into a handful of ultra-deep, well-sampled combined images in each of the six filters. We discuss in detail the methods used to calculate accurate transformations that provide optimal alignment of the input images, the methods used to perform sky background offsets in the input stack and the flagging of deviant pixels, and the balance reached between the input pixel drop size onto an output super-sampled pixel grid. Careful photometric, morphological, and astrometric measurements are performed on the stacks using iterative PSF-fitting techniques, and reveal unprecedented color-magnitude diagrams (CMDs) of the cluster extending to $>30$ th magnitude in the optical, 29 th magnitude in the UV, and 27th magnitude in the IR. The data set provides a characterization of the complete stellar populations of 47 Tuc, extending from the faintest hydrogen burning dwarfs through the main-sequence and giant branches, down to very cool white dwarf remnants in the cluster. The imaging also provides the deepest probe of the stellar populations of the background Small Magellanic Cloud (SMC) galaxy, resolving low mass main-sequence dwarfs with $M \lesssim 0.2 M_{\odot}$.

Subject headings: globular clusters: individual (47 Tucanae) - stars: evolution - stars: low-mass techniques: image processing - techniques: photometric - white dwarfs

${ }^{1}$ Based on observations with the NASA/ESA Hubble Space Telescope, obtained at the Space Telescope Science Institute, which is operated by the Association of Universities for Research in Astronomy, Inc., under NASA contract NAS5-26555. These observations are associated with proposal GO-11677.

2 Space Telescope Science Institute, 3700 San Martin Drive, Baltimore, MD, 21218; jkalirai@stsci.edu, jayander/dotter@stsci.edu

${ }^{3}$ Center for Astrophysical Sciences, Johns Hopkins University, Baltimore, MD, 21218

${ }^{4}$ Department of Physics \& Astronomy, University of British Columbia, Vancouver, BC, Canada; richer@astro.ubc.ca, kwoodley@phas.ubc.ca

${ }^{5}$ National Research Council, Herzberg Institute of Astrophysics, Victoria, BC, Canada; greg.fahlman/peter.stetson@nrc-cnrc.gc.ca

${ }^{6}$ Division of Astronomy and Astrophysics, University of California at Los Angeles, Los Angeles, CA, 90095; hansen/rmr@astro.ucla.edu, david.reitzel@gmail.com

${ }^{7}$ Center for Astrophysics \& Supercomputing, Swinburne University of Technology, Hawthorn VIC 3122, Australia; jhurley@swin.edu.au

${ }^{8}$ Department of Astronomy, Box 351580, University of Washington, Seattle, WA, 98195; king@astro.washington.edu

${ }^{9}$ Department of Astrophysics, American Museum of Natural History, Central Park West and 79th Street, New York, NY 10024; mshara@amnh.org

\section{INTRODUCTION}

Observations of the Milky Way's globular star clusters have represented one of the most important aspects of astronomy over the past century (Shapley 1917; Sandage 1953). Clusters are ideal testbeds for theories of stellar structure and evolution as well as dynamical processes, because their constituent stars share incredible similarities, being generally co-eval, co-spatial, and iso-metallic (Harris 1996; Vandenberg \& Bell 1985; Kalirai \& Richer 2010). Photometric measurements of the stars in any given cluster therefore provide a snapshot of how stellar and dynamical evolution operate at fixed conditions (e.g., age, metallicity, and cluster dynamical state), and this picture can be generalized by observing samples of clusters with different conditions. As one general application, modern day observations of nearby globular star clusters provide stringent tests of stellar-evolution models (e.g., Dotter et al. 2008), which in turn yield a calibration of population-synthesis techniques. These techniques are frequently used to interpret light from distant 
galaxies into fundamental properties such as star formation rates, chemical abundance trends, and mass-to-light ratios (e.g., Bruzual \& Charlot 2003).

In addition to being excellent tools for stellar structure and evolution theory, globular clusters also play an important role in our understanding of the epoch of baryonic structure formation in the Universe. Studies of star-forming regions in different environments frequently reveal sites of cluster formation (Whitmore \& Schweizer 1995; Hillenbrand 1997; Brodie \& Strader 2006), and therefore much observational and theoretical effort has been devoted to deriving accurate absolute ages of these systems. The most popular technique for this relies on the comparison of theoretical isochrones to the main-sequence turnoff morphology in the color-magnitude diagram (CMD), a feature that is dependent on nuclear reaction rates, uncertainties in chemical composition, the equation of state, and secondorder effects such as diffusion, rotation, and turbulence (Renzini \& Fusi Pecci 1988). Although the first measurements of the turnoff in a nearby globular cluster were made in the 1950s (Sandage 1953), a huge leap forward was enabled in the 1980s when CCDs came online and again recently by the high-resolution Advanced Camera for Surveys (ACS) on the Hubble Space Telescope (HST). For example, Sarajedini et al. (2007) executed a systematic and homogeneous survey of 65 Milky Way globular clusters and resolved the main-sequence turnoff of each of them with high photometric precision Anderson et al. 2008b). These data have led to a new understanding of the absolute ages and the age-metallicity relation of the Milky Way's globular-cluster population, and suggest that the most metal-poor clusters formed $\sim 1$ Gyr after the Big Bang (Hansen et al. 2007; Marín-Franch et al. 2009; Dotter et al. 2010).

Complementing the many ground- and space-based imaging programs that have studied both specific Milky Way globular clusters and the ensemble population, our team has been involved in a decade-long campaign to provide ultra-deep imaging of the complete stellar populations in the nearest clusters over multiple epochs. Our first study targeted the nearest globular cluster Messier 4 in 2001 (Cycle 9; GO-8679) with 123 orbits of HST observing time with the Wide Field Planetary Camera $2{ }^{10}$ and the second study targeted the second nearest cluster NGC 6397 in 2005 (Cycle 13; GO-10424) with 126 orbits of ACS imaging. These two projects have led to a wealth of scientific investigations that include 1) establishing accurate and independent white dwarf cooling ages for the clusters (Hansen et al. 2004; 2007), 2) constraints on the color-magnitude relation and luminosity and mass function of stars down to the lowest-mass dwarfs capable of core hydrogen burning (King et al. 1998; Richer et al. 2004; 2008), 3) new tests on the internal dynamical state of star clusters (Anderson et al. 2008a; Davis et al. 2008a; Hurley et al. 2008), 4) the most accurately constrained external space motions around the Milky Way through unprecedented proper motions (Kalirai et al. 2004; 2007; Anderson et al. 2008a), and 5) a wealth of variability and binary-related studies (Davis et al. 2008b).

Building on the success of these two previous programs,

\footnotetext{
10 Bedin et al. (2009) provide a more recent study of this cluster with ACS.
}

we received 121 orbits of $H S T$ observing time in Cycle 17 to establish a detailed imaging survey of one of the most luminous clusters in the sky, 47 Tucanae (GO-11677; PI - H. Richer). 47 Tuc is perhaps the best-studied globular cluster (e.g., Guhathakurta et al. 1992). Its rich stellar content has been targeted by large campaigns for studies of extrasolar planets (Gilliland et al. 2000), x-ray sources (Heinke et al. 2005), detailed dynamical studies (Meylan \& Mayor 1986; McLaughlin et al. 2006), and much more. The cluster is also the prototype metal-rich population in the Galactic halo and provides stringent tests for theories of stellar evolution (Demarque \& McClure 1977) and represents a metal-rich anchor for establishing star formation histories and abundances of resolved and unresolved stellar population in other galaxies (Brown et al. 2003; Bruzual \& Charlot 2003).

Unlike our previous studies of M4 and NGC 6397, this new project utilizes three $H S T$ cameras to image the cluster's stellar populations to unprecedented depth over both a wide wavelength range $(\lambda=0.4-1.7$ microns $)$ and over multiple fields spanning $>60 \operatorname{arcmin}^{2}$. This data set is aimed at achieving similar science goals to those listed above, yet over this expanded region of parameter space (e.g., the calibration of stellar-evolution models in the IR). The observations also yield the deepest probe to date of the lower main sequence of the Small Magellanic Cloud (SMC) dwarf galaxy, which is located behind 47 Tuc in projection (see Figure 1). The primary field is imaged at 13 orientations with the ACS/WFC in two visible filters $F 606 W$ and $F 814 W$ for all 121 orbits, and the parallel fields are mapped out by the newly installed Wide Field Camera 3 instrument using both the UVIS ( $F 390 W$ and $F 606 W)$ and IR $(F 110 W$ and $F 160 W)$ cameras. The wavelength coverage of these filters is shown relative to the spectral energy distribution of three stars that are relevant to the present science program in Figure 3, a hot white dwarf, a moderate temperature globular cluster turnoff star, and a cool M dwarf.

To support the many on-going scientific projects enabled by this data set, we describe here the detailed observational strategy and data-reduction methods that are used to combine over 700 individual full-frame images into a single well-sampled stack in each filter which is largely free of cosmetic defects. The methods that are used to measure the photometry, astrometry, and morphology of all stellar sources on these combined images are also described. The final CMDs exhibit well defined sequences extending down to 29th magnitude in the UV, 30 th magnitude in the visible, and 27th magnitude in the IR, and the photometric and astrometric accuracy is assessed using an extensive set of artificial-star tests. The primary features of these CMDs are illustrated in relation to the science goals. The programs used for image registration, combination, and photometry in this analysis are all freely available and therefore some of the specific techniques used here may be useful for other large $H S T$ programs.

\section{OBSERVATIONAL DESIGN}

The observational design of this program was constructed to specifically address several independent scientific questions. The most important consideration was to establish a photometric catalog in two filters that provides accurate characterization of the faintest white 


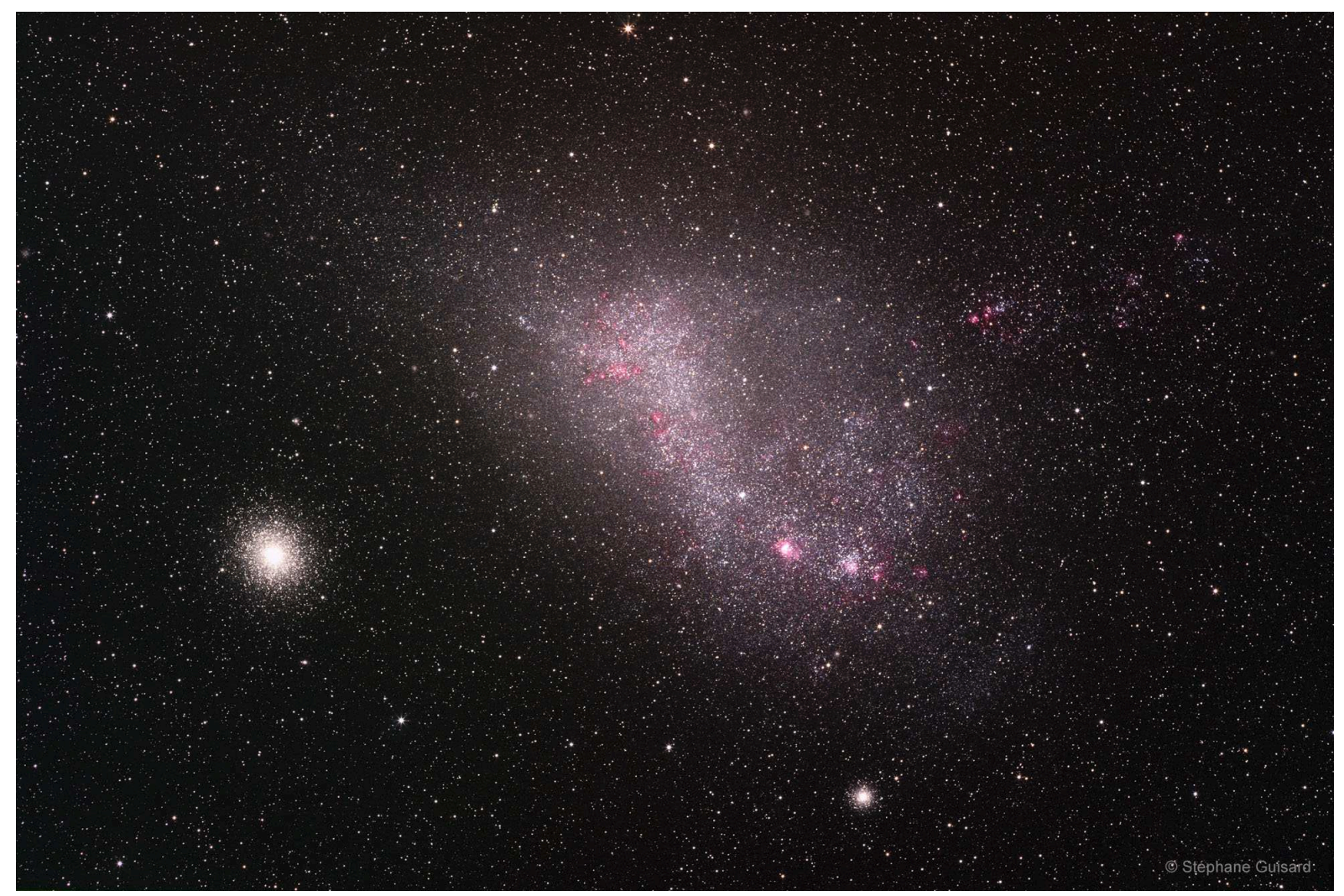

FIG. 1.- A wide-field ground-based image of the Small Magellanic Cloud (SMC) in the southern skies reveals two foreground Milky Way globular clusters, NGC 362 just below the SMC and 47 Tuc to the left of the galaxy. Although the main body of the SMC is separated from 47 Tuc by more than two degrees, a diffuse stellar population persists to greater radii and represents a background source of stars in our study (as demonstrated later). This image subtends $6.8 \times 4.5$ degrees and was taken with a $300 \mathrm{~mm}$ lens in September 2007. The image was made by combining multiple 10 minute exposures in five visible filters (including H $\alpha$ ). Image is courtesy of Stéphane Guisard and reproduced here with permission, http://www.astrosurf.com/sguisard

dwarfs in 47 Tuc (i.e., for an independent age measurement). Based both on white dwarf cooling models for low masses and our previous experience from the deep studies of M4 and NGC 6397 (Hansen et al. 2004, 2007), the white dwarf luminosity function is expected to span over 5 magnitudes in the CMD and be most efficiently measured using observations at visible wavelengths. The secondary science goals require mapping the color-magnitude relation and stellar mass function of stars down to the faintest dwarfs on the main-sequence. These stars are cooler than the faintest white dwarfs and have redder colors, suggesting observations in the near IR are needed. A third science goal requires establishing a panchromatic data set for the cluster's stellar populations. This will enable a sensitive study of the stellar main-sequence and turnoff by stretching out features in the CMD and providing enhanced sensitivity to any splittings or multiple sequences, and also provide tests of the spectral energy distributions of exotic stellar populations (e.g., CVs, blue stragglers, white dwarfs with accretion disks, etc.). Finally, we require sampling the cluster population at a range of radii to test dynamical models of cluster formation and evolution.

To satisfy these requirements, the design of the program used three HST cameras on two instruments operating simultaneously, ACS, WFC3/UVIS, and
WFC3/IR. The primary field was intended to reach the faintest magnitudes and requires well-sampled images that are free of image defects caused by diffraction spikes, cosmic rays, bad pixels, and charge transfer efficiency (CTE) trails. The design for the parallel fields followed from the primary observations, and achieved greater sensitivity to the radial distribution of the stellar population by imaging a larger field of view. This was achieved by rolling the telescope around the primary field multiple times, such that the secondary instrument swept out a contiguous arc. This design is highlighted in Figure 2 through a spatial map of the primary and parallel $H S T$ imaging fields superimposed on a ground based image of 47 Tuc. In the following subsections, we first justify and describe the ACS and WFC3 observations in detail, with logs of the exposures obtained with all three cameras. A summary of the overall observational plan is then provided in Section 2.3. which also describes this visualization in more detail.

\subsection{The Primary Field - Advanced Camera for Surveys}

We chose ACS/WFC as the instrument for the deep primary imaging field. ACS offers a larger field of view than WFC3/UVIS and slightly better sensitivity at redder wavelengths. Observations with ACS can also be more easily compared with our previous study of 

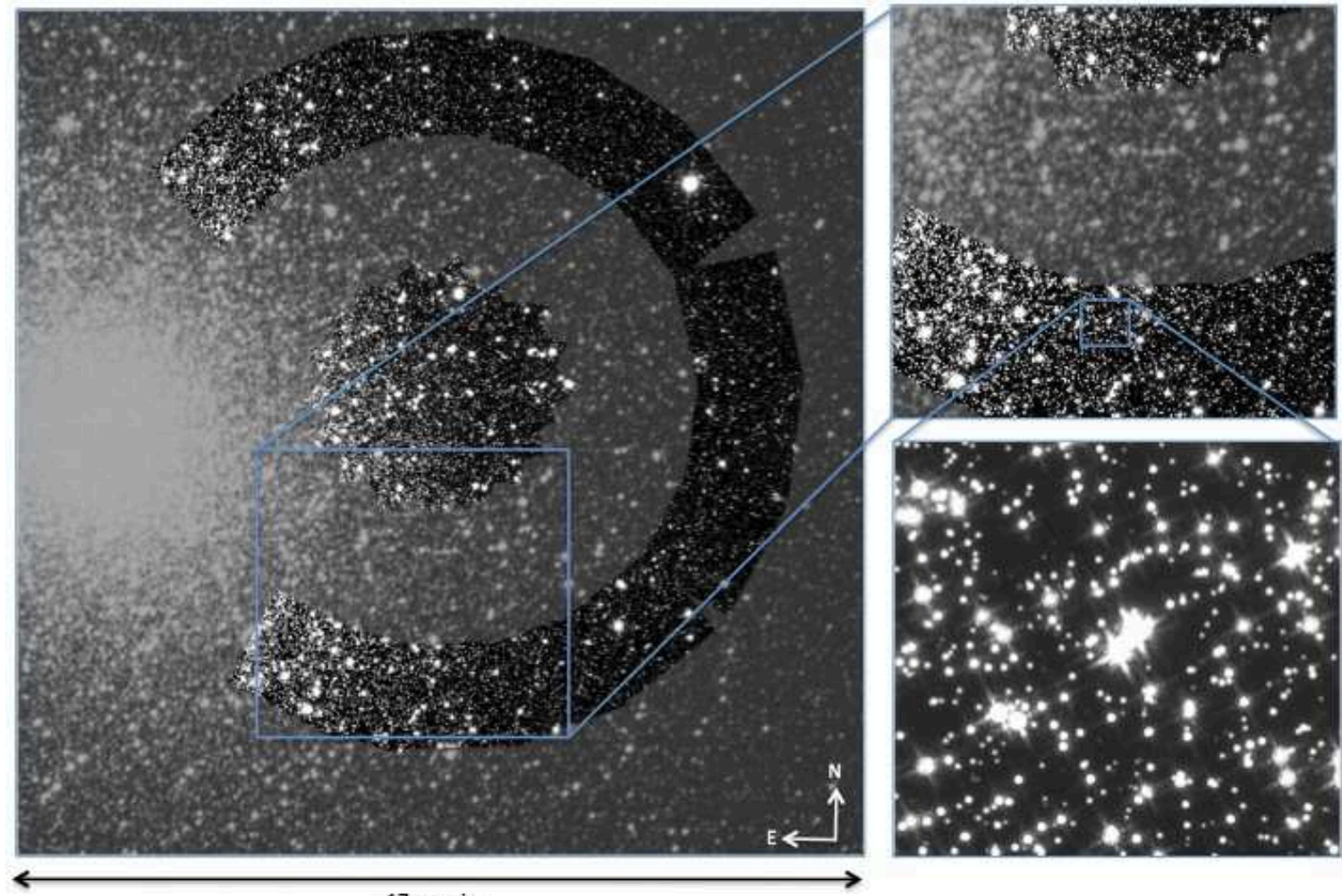

$17 \operatorname{arcmin}$

FIG. 2.- A visualization of the observational strategy which uses three HST cameras. The picture in the backdrop is a ground based Digital Sky Survey image of 47 Tuc, resampled to a scale of 0.1 arcsec per pixel. The star-like pointing to the west of the cluster center in the center of the mosaic represents the deep ACS/WFC field, which was imaged in $F 606 W$ and $F 814 W$ for all 121 orbits of the program. The pattern is caused by the requirement to roll the telescope by $\sim 20$ degree in half of the visits. These multiple roll angles provide very efficient cleaning of image defects and artifacts, and also allow the secondary WFC3 instrument to sweep out an arc around the periphery of the cluster. These parallel fields sample more than $60 \mathrm{arcmin}^{2}$ of the cluster's population from $R=6.5-17.9$ pc with both the WFC3/UVIS camera in $F 390 W$ and $F 606 W$ and the WFC3/IR camera in the $F 110 W$ and $F 160 W$ filters. The two panels on the right show zoomed regions of a small portion of the parallel WFC3 fields. This mosaic was constructed by drizzling each pixel in the HST program onto the ground based Digital Sky Survey image, where WFC3/IR F160W was arbitrarily selected for the parallel fields.

NGC 6397 and with previous epoch 47 Tuc images for proper-motion analysis. The wide-band $F 606 \mathrm{~W}$ filter was chosen as the primary spectral element given its excellent throughput and a central wavelength that is well matched to the peak in the spectral energy distribution of cool white dwarfs. For the second filter, the wideband $F 814 W$ spectral element was chosen as it also provides excellent throughput and redder sensitivity for secondary science goals related to mapping the low mass main-sequence and red dwarf population of the cluster.

White dwarfs with $\mathrm{M}=0.5 M_{\odot}$ dim to $M_{F 606 W} \sim 16$ after 12 Gyr (Hansen et al. 2007), translating to a faint observed visual magnitude of $\sim 29.3$ at the distance of 47 Tuc (4.2 kpc; Zoccali et al. 2001). We needed 121 orbits of exposure time in a single deep field to definitively measure this limit in the white dwarf cooling sequence. The exposure time was split between 117 exposures in $F 606 W$ and 125 deep exposures in $F 814 W$. The exposure times of the $F 606 \mathrm{~W}$ frames ranged from 1113 - 1498 seconds for a total integration of $163.7 \mathrm{ks}$ and those in $F 814 W$ ranged from 1031 - 1484 seconds for an integration of $172.8 \mathrm{ks}$. Additional exposures of shorter lengths were also obtained to measure bright stars, which are saturated on the deep frames. We obtained $4 \times 100 \mathrm{~s}$, $4 \times 10 \mathrm{~s}$, and $4 \times 1 \mathrm{~s}$ exposures in $F 606 W$ and $5 \times 100 \mathrm{~s}$, $4 \times 10 \mathrm{~s}$, and $4 \times 1 \mathrm{~s}$ in $F 814 \mathrm{~W}$.

Sources on individual HST images are affected by cosmic rays, hot pixels, CTE losses, diffraction spikes from bright saturated stars, possible intrapixel sensitivity variations, and other pixel-to-pixel effects from errors in the flat field and geometric distortion. In order to mitigate these issues, we chose to observe the scene at different dither positions and at different roll angles so that a given source would be observed in many different pixels at many different places on the detector. This dithering also minimizes errors caused by uncertainties in the pixelto-pixel flat fielding and intra-pixel sensitivities. The observational strategy yielded two deep images with ACS in each orbit, with five orbits being grouped into a visit. Within each visit, the observations were dithered in both the $x$ and $y$ directions. The $y$ dither ensured that no star would fall in the gap between the WFC1 and WFC3 chips on ACS on more than one observation through the same filter. For visits taken at the same orientation, a small 
$x$ dither ensured that stars would land in different pixels in the different visits. This dither pattern provides easy discrimination of bad pixels. Artifacts caused by diffraction spikes from the telescope support structure, bleeding of saturated pixels into neighboring regions, and CTE losses are more difficult to deal with. These artifacts are aligned with a particular direction and correlated with the position of the source relative to the amplifier that reads out the charge, therefore a linear dither pattern will not result in efficient recovery of information. To solve this problem, we constructed the observations such that 13 of the 24 visits and their corresponding orbits were executed at 13 specific roll angles separated by $\delta$ $\sim 20$ degrees. The locations of stars on the images and their respective read out positions are therefore effectively randomized in the field of view. The remaining 12 fields were imaged at a much smaller range in roll angle (within \pm 5 degree), largely because of $H S T$ scheduling constraints and also to ensure a deeper parallel field at fixed location (see below). An exposure time weighted map of these primary field observations is shown in Figure 4

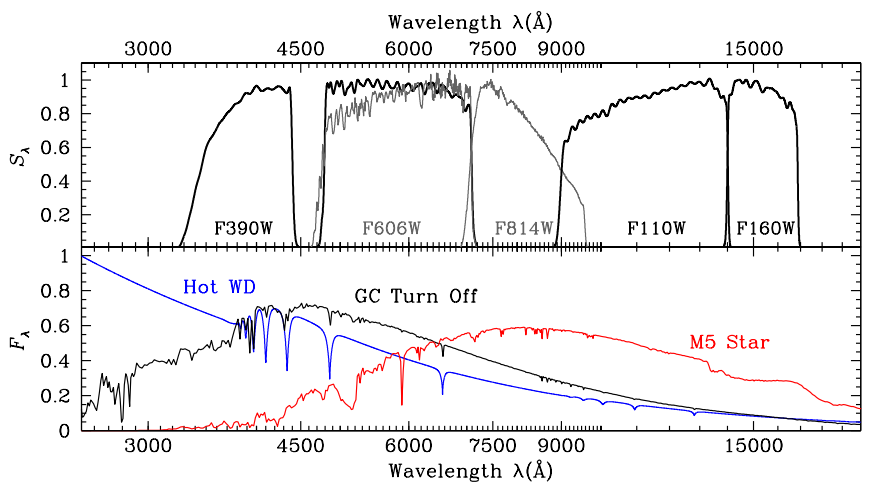

FIG. 3.- The wavelength coverage of the ACS (grey), WFC3/UVIS (black), and WFC/IR (black) filters are shown relative to the spectral energy distribution of three different stars that are among the target populations. These include a hot white dwarf star (blue), a typical globular cluster turnoff star (black), and a cool M dwarf (red). The WFC3/UVIS F390W filter provides superior throughput compared to bluer filters on the instrument, and is ideally suited for 47 Tuc observations given the lack of a hot horizontal branch in the cluster. Note, that the $F 606 W$ filter was used on both ACS (grey) and WFC3/UVIS (black).

The location of the primary ACS field within 47 Tuc was chosen based on several factors. The cluster is located $4.2 \mathrm{kpc}$ from the Sun and spans a large angular size $\left(r_{t}=42 \operatorname{arcmin}=51 \mathrm{pc}\right)$. We require a field that is neither too sparse nor too crowded such that an appreciable fraction of faint stars near the termination of the white dwarf cooling sequence at $\sim 29$ th magnitude can be isolated and measured. We also prefer a field where dynamical models suggest that the correction from the observed cluster mass function to the global mass function is small, which occurs at $\sim 2$ half mass radii according to Hurley et al. (2008). Finally, the field needed to overlap previous epoch imaging observations to permit proper-motion separation of the cluster stars from the field stars. All of these requirements were satisfied by centering the ACS primary observations at $\alpha=00: 22: 39$, $\delta=-72: 04: 04$, which is located about $6.7 \operatorname{arcmin}(8.8 \mathrm{pc})$ west of the cluster center. This calibration field has been

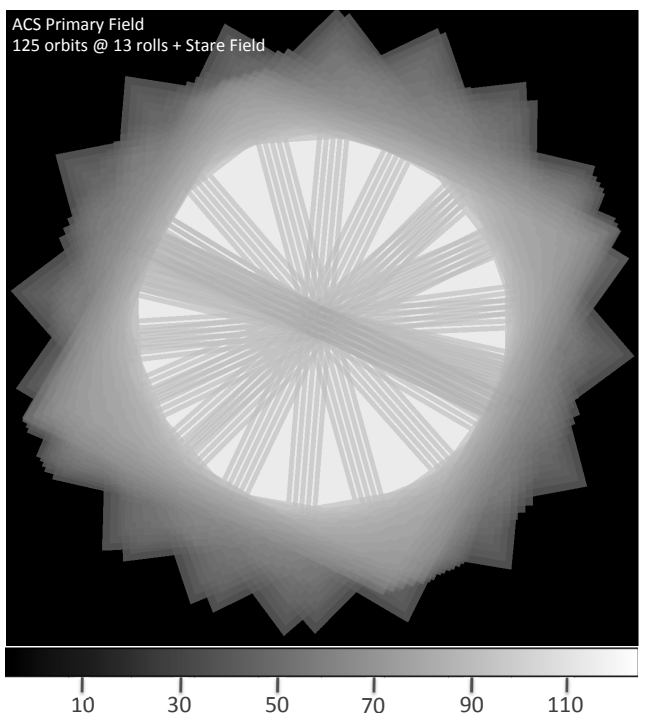

Fig. 4. - An exposure time map of the primary ACS field for all $125 \mathrm{~F} 814 \mathrm{~W}$ images, including the 58 images at an approximately fixed roll angle of $\sim 293$ degrees in the Stare field. The star-like pattern is generated through the multiple roll angles of the observations. The color scale gives an indication of the number of images in the stack, where the average exposure time of each of the images was 1382 seconds. The bulk of the field of view has very nice depth of $>150$ ks (also in $F 606 W$ ), and because of the multiple dithers and roll angles, the actual sky coverage is $\sim 50 \%$ larger than the ACS detector's field of view.

observed more than 245 times in $F 606 \mathrm{~W}$ and 66 times in $F 814 W$ over the lifetime of ACS.

\subsection{The Parallel Fields - Wide Field Camera 3}

The newly installed WFC3 instrument provides a nice complement to ACS by offering a second HST imager that operates at high resolution and has a large field of view. The instrument contains two cameras that are optimized for UV/visible wavelengths (UVIS) and for IR wavelengths (IR). Whereas previous generation HST instruments such as NICMOS were frequently used to study distant galaxies, the field of view limited what could be done in nearby resolved stellar populations with a large angular extent. To date, very few deep CMDs with high precision photometry exist in the IR bandpasses, and tests of stellar evolution theory in this spectral range are almost non-existent.

Several aspects of the parallel WFC3 observations were automatically set by the primary ACS design. The angular separation of the ACS and WFC3 instruments is $\sim 6^{\prime}$, so the 13 different roll angles of the primary field map to 13 separate parallel pointings. The $\sim 20$ degree step size in the roll angle between each of the primary field observations also ensured that the parallel fields form a contiguous imaging region with the WFC3/IR camera (smaller field of view than WFC3/UVIS - $4.6 \operatorname{arcmin}^{2}$ ). The telescope orientation was chosen such that the parallel fields swept out an arc to the west of the primary pointing, away from the dense region near the cluster center. This arc extends 250 degrees in azimuth and over a radial range of $5-13.7 \operatorname{arcmin}(6.5-17.9 \mathrm{pc})$, thereby sampling the cluster population over a large dynamical range (47 Tuc's core radius is 0.36 arcmin). The total area of the cluster imaged in these pointings is $>60$ sq arcmin with WFC3/IR (larger with WFC3/UVIS). In referring to the WFC3 parallel fields from here on, the 
TABLE 1

The Observational Log of the ACS/WFC Associations ${ }^{1}$

\begin{tabular}{|c|c|c|c|c|c|c|c|}
\hline Dataset Name & Visit & Obs. Date & Filter & $\begin{array}{c}\mathrm{RA} \\
(\mathrm{J} 2000)\end{array}$ & $\begin{array}{c}\mathrm{DEC} \\
(\mathrm{J} 2000)\end{array}$ & $\begin{array}{c}\text { Exp. Time } \\
\text { (s) }\end{array}$ & $\begin{array}{c}\text { Orientation } \\
\left({ }^{\circ} \mathrm{N} \text { of } \mathrm{E}\right)\end{array}$ \\
\hline JB6V01010 & 01 & $2010-02-13$ & F606W & $00: 22: 36.35$ & $-72: 04: 04.4$ & 6920.0 & 316.020 \\
\hline JB6V01020 & 01 & 2010-02-13 & F814W & $00: 22: 36.35$ & $-72: 04: 04.4$ & 7269.0 & 316.020 \\
\hline JB6V02010 & 02 & 2010-03-04 & F814W & $00: 22: 36.47$ & $-72: 04: 00.7$ & 6921.0 & 336.126 \\
\hline JB6V02020 & 02 & 2010-03-04 & F606W & $00: 22: 36.47$ & $-72: 04: 00.7$ & 7323.0 & 336.126 \\
\hline JB6V03010 & 03 & $2010-03-16$ & F606W & $00: 22: 36.80$ & $-72: 03: 57.8$ & 6809.0 & 354.209 \\
\hline JB6V03020 & 03 & 2010-03-16 & $\mathrm{F} 814 \mathrm{~W}$ & $00: 22: 36.80$ & $-72: 03: 57.8$ & 7089.0 & 354.209 \\
\hline JB6V04010 & 04 & 2010-04-10 & $\mathrm{F} 814 \mathrm{~W}$ & $00: 22: 39.23$ & $-72: 04: 08.7$ & 6930.0 & 17.276 \\
\hline JB6V04020 & 04 & 2010-04-10 & F606W & $00: 22: 39.23$ & $-72: 04: 08.7$ & 7315.0 & 17.276 \\
\hline JB6V25010 & 25 & $2010-05-03$ & F606W & $00: 22: 38.27$ & $-72: 03: 53.6$ & 6961.0 & 38.312 \\
\hline JB6V25020 & 25 & 2010-05-03 & $\mathrm{F} 814 \mathrm{~W}$ & $00: 22: 38.27$ & $-72: 03: 53.6$ & 7269.0 & 38.312 \\
\hline JB6V06010 & 06 & $2010-06-12$ & F814W & $00: 22: 39.07$ & $-72: 03: 53.7$ & 6512.0 & 58.301 \\
\hline JB6V06020 & 06 & $2010-06-12$ & F606W & $00: 22: 39.07$ & $-72: 03: 53.7$ & 7023.0 & 58.301 \\
\hline JB6V07010 & 07 & $2010-06-18$ & F606W & $00: 22: 40.02$ & $-72: 03: 55.6$ & 6920.0 & 84.233 \\
\hline JB6V07020 & 07 & 2010-06-18 & F814W & $00: 22: 40.02$ & $-72: 03: 55.6$ & 7269.0 & 84.233 \\
\hline JB6V08010 & 08 & 2010-07-29 & $\mathrm{F} 814 \mathrm{~W}$ & $00: 22: 40.52$ & $-72: 03: 57.9$ & 8969.0 & 102.157 \\
\hline JB6V08020 & 08 & $2010-07-29$ & F606W & $00: 22: 37.71$ & $-72: 04: 07.2$ & 4125.0 & 102.168 \\
\hline JB6V09010 & 09 & $2010-08-05$ & F606W & $00: 22: 40.84$ & $-72: 04: 00.9$ & 6929.0 & 120.066 \\
\hline JB6V09020 & 09 & 2010-08-05 & F814W & $00: 22: 40.84$ & $-72: 04: 00.9$ & 7269.0 & 120.066 \\
\hline JB6V10010 & 10 & 2010-08-14 & $\mathrm{F} 814 \mathrm{~W}$ & $00: 22: 40.94$ & $-72: 04: 04.6$ & 6930.0 & 139.959 \\
\hline JB6V10020 & 10 & $2010-08-14$ & F606W & $00: 22: 40.94$ & $-72: 04: 04.6$ & 7323.0 & 139.959 \\
\hline JB6V11010 & 11 & 2010-09-19 & F606W & $00: 22: 40.79$ & $-72: 04: 08.0$ & 6960.0 & 158.861 \\
\hline JB6V11020 & 11 & 2010-09-19 & $\mathrm{F} 814 \mathrm{~W}$ & $00: 22: 40.79$ & $-72: 04: 08.0$ & 7269.0 & 158.861 \\
\hline JB6V12010 & 12 & 2010-10-01 & $\mathrm{F} 814 \mathrm{~W}$ & $00: 22: 40.40$ & $-72: 04: 11.0$ & 6961.0 & 177.777 \\
\hline JB6V12020 & 12 & $2010-10-01$ & F606W & $00: 22: 40.40$ & $-72: 04: 11.0$ & 7323.0 & 177.777 \\
\hline JB6V13010 & 13 & $2010-01-16$ & F606W & $00: 22: 36.70$ & $-72: 04: 08.9$ & 6791.0 & 287.368 \\
\hline JB6V13020 & 13 & 2010-01-16 & $\mathrm{F} 814 \mathrm{~W}$ & $00: 22: 36.70$ & $-72: 04: 08.9$ & 7014.0 & 287.368 \\
\hline JB6V14010 & 14 & $2010-01-17$ & $\mathrm{~F} 814 \mathrm{~W}$ & $00: 22: 36.67$ & $-72: 04: 09.2$ & 6707.0 & 287.368 \\
\hline JB6V14020 & 14 & $2010-01-17$ & F606W & $00: 22: 36.67$ & $-72: 04: 09.2$ & 7153.0 & 287.368 \\
\hline JB6V15010 & 15 & $2010-01-18$ & F606W & $00: 22: 36.66$ & $-72: 04: 09.5$ & 6791.0 & 287.368 \\
\hline JB6V15020 & 15 & $2010-01-18$ & F814W & $00: 22: 36.66$ & $-72: 04: 09.5$ & 7014.0 & 287.368 \\
\hline JB6V16010 & 16 & 2010-01-19 & F814W & $00: 22: 36.63$ & $-72: 04: 09.7$ & 6707.0 & 287.688 \\
\hline JB6V16020 & 16 & 2010-01-19 & F606W & $00: 22: 36.63$ & $-72: 04: 09.7$ & 7153.0 & 287.688 \\
\hline JB6V17010 & 17 & $2010-01-20$ & F606W & $00: 22: 36.62$ & $-72: 04: 08.7$ & 6791.0 & 288.829 \\
\hline JB6V17020 & 17 & $2010-01-20$ & F814W & $00: 22: 36.62$ & $-72: 04: 08.7$ & 7014.0 & 288.829 \\
\hline JB6V18010 & 18 & $2010-01-20$ & F814W & $00: 22: 36.57$ & $-72: 04: 08.8$ & 6721.0 & 289.892 \\
\hline JB6V18020 & 18 & $2010-01-21$ & F606W & $00: 22: 36.57$ & $-72: 04: 08.8$ & 7139.0 & 289.892 \\
\hline JB6V19010 & 19 & 2010-01-25 & F606W & $00: 22: 36.47$ & $-72: 04: 08.2$ & 6791.0 & 294.706 \\
\hline JB6V19020 & 19 & $2010-01-25$ & F814W & $00: 22: 36.47$ & $-72: 04: 08.2$ & 7014.0 & 294.706 \\
\hline JB6V20010 & 20 & $2010-01-23$ & F814W & $00: 22: 36.57$ & $-72: 04: 09.7$ & 9590.0 & 287.871 \\
\hline JB6V20020 & 20 & $2010-01-23$ & F606W & $00: 22: 38.56$ & $-72: 04: 04.5$ & 4270.0 & 287.863 \\
\hline JB6V21010 & 21 & 2010-01-26 & F606W & $00: 22: 36.62$ & $-72: 04: 07.7$ & 6791.0 & 295.702 \\
\hline JB6V21020 & 21 & $2010-01-26$ & $\mathrm{~F} 814 \mathrm{~W}$ & $00: 22: 36.62$ & $-72: 04: 07.7$ & 7014.0 & 295.702 \\
\hline JB6V22010 & 22 & $2010-01-27$ & F814W & $00: 22: 36.57$ & $-72: 04: 07.7$ & 6707.0 & 296.852 \\
\hline JB6V22020 & 22 & $2010-01-27$ & F606W & $00: 22: 36.57$ & $-72: 04: 07.7$ & 7153.0 & 296.852 \\
\hline JB6V23010 & 23 & 2010-01-28 & F606W & $00: 22: 36.53$ & $-72: 04: 07.8$ & 6791.0 & 298.002 \\
\hline JB6V23020 & 23 & $2010-01-28$ & $\mathrm{~F} 814 \mathrm{~W}$ & $00: 22: 36.53$ & $-72: 04: 07.8$ & 7014.0 & 298.002 \\
\hline JB6V24010 & 24 & $2010-01-15$ & F814W & $00: 22: 36.69$ & $-72: 04: 09.8$ & 8064.0 & 287.368 \\
\hline JB6V24020 & 24 & $2010-01-15$ & F606W & $00: 22: 36.69$ & $-72: 04: 09.8$ & 8596.0 & 287.368 \\
\hline
\end{tabular}

${ }^{a}$ The observational log is grouped into $H S T$ visits, where each visit consists of five orbits spread over the two filters (except Visit 24 which has six orbits) and each orbit consists of two deep exposures and occasional shorter exposures.

${ }^{b}$ The total integration time for all deep and short observations.

deep field with the \pm 5 degree fixed roll angle are labelled as the "Stare" field (observed for 61 orbits) and the 12 other fields as the "Swath" fields (observed for 5 orbits each).

The science goals in the program require imaging the stellar populations in the Swath and Stare fields both at UV and IR wavelengths. The separate data sets in each of these regimes are very valuable. For example, the UV observations over this wide field of view will provide important constraints on the luminosity function of the brightest white dwarfs that cool via neutrino emission, and also provide deep imaging for counterpart studies to exotic stellar populations such as LMXBs and CVs. The IR data are intended to completely characterize the 47 Tuc main-sequence from the brightest giants to the reddest dwarfs near the hydrogen burning limit. Taken together, this imaging also provides matched catalogs of stars measured over the full wavelength baseline from UV through the optical to IR.

The WFC3 observations were split over four wideband filters. For the UVIS camera, the F390W and $F 606 W$ filters were chosen, and two long dithered exposures ( $\gtrsim 1200 \mathrm{~s})$ were obtained in a single orbit for each of the two filters. The $F 390 W$ filter was picked over bluer UV filters on WFC3 for two main reasons. First, 47 Tuc is a metal-rich globular cluster with $[\mathrm{Fe} / \mathrm{H}]$ $=-0.70$ (Carretta et al. 2009) and does not contain an extended blue horizontal branch. Second, the exposure times in each WFC3/UVIS field were fairly short and therefore we prefered $F 390 W$ for its substantially bet- 
TABLE 2

The Observational Log of the WFC3/UVis Associations ${ }^{1}$

\begin{tabular}{|c|c|c|c|c|c|c|c|}
\hline Dataset Name & Visit & Obs. Date & Filter & $\begin{array}{c}\mathrm{RA} \\
(\mathrm{J} 2000)\end{array}$ & $\begin{array}{c}\text { DEC } \\
(\mathrm{J} 2000)\end{array}$ & $\begin{array}{l}\text { Exp. } \text { Time }^{2} \\
(\mathrm{~s})\end{array}$ & $\begin{array}{c}\text { Orientation } \\
\left({ }^{\circ} \mathrm{N} \text { of } \mathrm{E}\right)\end{array}$ \\
\hline \multicolumn{8}{|l|}{ Swath fields } \\
\hline IB6V01031 & 01 & $2010-02-13$ & F390W & $00: 22: 34.47$ & $-72: 09: 58.8$ & 2661.0 & 316.020 \\
\hline IB6V01051 & 01 & $2010-02-13$ & F606W & $00: 22: 34.47$ & $-72: 09: 58.8$ & 2849.0 & 316.020 \\
\hline IB6V02031 & 02 & 2010-03-04 & F390W & $00: 22: 08.32$ & $-72: 09: 30.7$ & 2658.0 & 336.126 \\
\hline IB6V02051 & 02 & 2010-03-04 & F606W & $00: 22: 08.32$ & $-72: 09: 30.7$ & 2849.0 & 336.126 \\
\hline IB6V03031 & 03 & $2010-03-16$ & F390W & $00: 21: 47.88$ & $-72: 08: 31.4$ & 2606.0 & 354.209 \\
\hline IB6V03051 & 03 & 2010-03-16 & F606W & $00: 21: 47.88$ & $-72: 08: 31.4$ & 2771.0 & 354.209 \\
\hline IB6V04031 & 04 & 2010-04-10 & F390W & $00: 21: 29.30$ & $-72: 06: 38.5$ & 2667.0 & 17.283 \\
\hline IB6V04051 & 04 & $2010-04-10$ & F606W & $00: 21: 29.30$ & $-72: 06: 38.5$ & 2845.0 & 17.283 \\
\hline IB6V25031 & 25 & 2010-05-03 & F390W & $00: 21: 21.98$ & $-72: 04: 33.5$ & 2699.0 & 38.312 \\
\hline IB6V25051 & 25 & 2010-05-03 & F606W & $00: 21: 21.98$ & $-72: 04: 33.5$ & 2849.0 & 38.312 \\
\hline IB6V06031 & 06 & 2010-06-12 & F390W & $00: 21: 24.50$ & $-72: 02: 30.7$ & 2548.0 & 58.301 \\
\hline IB6V06051 & 06 & 2010-06-12 & F606W & $00: 21: 24.50$ & $-72: 02: 30.7$ & 2699.0 & 58.301 \\
\hline IB6V07031 & 07 & $2010-06-18$ & F390W & $00: 21: 40.90$ & $-72: 00: 10.0$ & 2657.0 & 84.233 \\
\hline IB6V07051 & 07 & $2010-06-18$ & F606W & $00: 21: 40.90$ & $-72: 00: 10.0$ & 2849.0 & 84.233 \\
\hline IB6V08031 & 08 & $2010-07-29$ & F390W & $00: 21: 59.33$ & $-71: 58: 59.0$ & 2426.0 & 102.157 \\
\hline IB6V08051 & 08 & 2010-07-29 & F606W & $00: 21: 59.33$ & $-71: 58: 59.0$ & 2619.0 & 102.157 \\
\hline IB6V09031 & 09 & 2010-08-05 & F390W & $00: 22: 21.57$ & $-71: 58: 17.8$ & 2666.0 & 120.066 \\
\hline IB6V09051 & 09 & 2010-08-05 & F606W & $00: 22: 21.57$ & $-71: 58: 17.8$ & 2849.0 & 120.066 \\
\hline IB6V10031 & 10 & $2010-08-14$ & F390W & $00: 22: 48.11$ & $-71: 58: 11.6$ & 2666.0 & 139.959 \\
\hline IB6V10051 & 10 & $2010-08-14$ & F606W & $00: 22: 48.11$ & $-71: 58: 11.6$ & 2849.0 & 139.959 \\
\hline IB6V11031 & 11 & 2010-09-19 & F390W & $00: 23: 12.33$ & $-71: 58: 44.9$ & 2699.0 & 158.861 \\
\hline IB6V11051 & 11 & 2010-09-19 & F606W & $00: 23: 12.33$ & $-71: 58: 44.9$ & 2849.0 & 158.861 \\
\hline IB6V12031 & 12 & $2010-10-01$ & F390W & $00: 23: 32.93$ & $-71: 59: 52.9$ & 2699.0 & 177.777 \\
\hline IB6V12051 & 12 & $2010-10-01$ & F606W & $00: 23: 32.93$ & $-71: 59: 52.9$ & 2849.0 & 177.777 \\
\hline $\begin{array}{l}\text { Stare field } \\
\text { IB6V13031 }\end{array}$ & 13 & 2010-01-16 & F390W & $00 \cdot 23 \cdot 11$ & $-72 \cdot 0$ & 26140 & 287368 \\
\hline IB6V13051 & 13 & $2010-01-16$ & F606W & $00: 23: 11.84$ & $-72: 09: 24.1$ & 2764.0 & 287.368 \\
\hline
\end{tabular}

${ }^{a}$ The observational $\log$ is grouped into HST visits, where each visit with WFC3/UVIS consists of two orbits (one per filter) and each orbit consists of two deep and two short exposures.

${ }^{b}$ The total integration time for all deep and short observations.

ter throughput compared to $F 336 W, F 275 W$, and other UV filters. The choice of $F 606 W$ was made to ensure a high throughput in these shallow observations and to provide a visible band measurement for the panchromatic study. To characterize the brighter stars in the UV data, two dithered $50 \mathrm{~s}$ exposures in each of the $F 390 \mathrm{~W}$ and $F 606 W$ filters were also obtained, for each of the 13 fields. The total exposure time with WFC3/UVIS in the program was $34.3 \mathrm{ks}$ for the $52 \mathrm{~F} 390 \mathrm{~W}$ images and $36.5 \mathrm{ks}$ for the $52 \mathrm{~F} 606 \mathrm{~W}$ images (only $1300 \mathrm{~s}$ total came from the shallower $50 \mathrm{~s}$ exposures in each filter). As demonstrated later, a single orbit's observations in each of these filters (in each of the fields) with WFC3/UVIS provides characterization of 47 Tuc stars down to $>28$ th magnitude.

For the IR camera, the two widely used wide-band filters $F 110 W$ and $F 160 W$ were picked for their high throughput and sampling of the full available IR wavelength range. In each of the 12 Swath fields, two dithered exposures with $F 110 W$ of length 1200 - 1400 s were obtained in a single orbit (total integration of $33.3 \mathrm{ks}$ for the 24 images) and four dithered exposures with $F 160 \mathrm{~W}$ of length $1200-1400 \mathrm{~s}$ were obtained in two orbits (total integration of $57.6 \mathrm{ks}$ for the 48 images). An equal number of images with shorter exposure times of $100-350$ s were also obtained in each field $(3.2 \mathrm{ks}$ in $F 110 W$ and $13.7 \mathrm{ks}$ in $F 160 W$ ). Unlike for the WFC3/UVIS observations, the Stare field was treated differently for WFC3/IR by investing a substantial integration to achieve a deeper, well sampled image in each IR filter. Altogether, 38 im- ages in $F 110 W$ were collected with individual integration times of $1200-1400 \mathrm{~s}(51.2 \mathrm{ks}$ total $)$ and 80 images in $F 160 W$ were collected with integration times of $1200-$ $1400 \mathrm{~s}$ (103.9 ks total). Shallower exposures of $32-300 \mathrm{~s}$ were also obtained and total $4.1 \mathrm{ks}$ in $F 110 W$ and $10.4 \mathrm{ks}$ in $F 160 \mathrm{~W}$. The combined Stare field observations with WFC3/IR total 59 orbits alone.

\subsection{Summary of Observations}

Given the complex design with multiple roll-angle restrictions, the observations described above were collected over multiple epochs extending from January to October 2010. In total we obtained 708 full frame images with ACS, WFC3/UVIS, and WFC3/IR with a total exposure time of $0.75 \mathrm{Ms}$. In the next section, we discuss an analysis of these data that reduces the complete data set to a set of single images in each filter for the deep and shallow observations.

In Figure 2, a visualization of these $H S T$ data was presented. This figure illustrates a montage of the ACS primary field and the WFC3/IR Stare and Swath fields, superimposed within a ground-based Digital Sky Survey image of 47 Tuc. The ground-based image in the background has been resampled to 0.1 arcsec / pixel and subtends an angular scale of $\sim 16.7$ arcmin (i.e., 10,000 pixels $\times 10,000$ pixels). The positions and fluxes of all sources on this image were measured to produce a catalog, and astrometrically aligned using 2MASS standards. This master astrometric grid was first used to calculate transformations between each of the new HST images and the catalog, and then every pixel of every deep image that 
TABLE 3

The Observational Log of the WFC3/IR Associations ${ }^{1}$

\begin{tabular}{|c|c|c|c|c|c|c|c|}
\hline Dataset Name & Visit & Obs. Date & Filter & $\begin{array}{c}\mathrm{RA} \\
(\mathrm{J} 2000)\end{array}$ & $\begin{array}{c}\text { DEC } \\
(\mathrm{J} 2000)\end{array}$ & $\begin{array}{c}\text { Exp. } \text { Time }^{2} \\
(\mathrm{~s})\end{array}$ & $\begin{array}{c}\text { Orientation } \\
\left({ }^{\circ} \mathrm{N} \text { of } \mathrm{E}\right)\end{array}$ \\
\hline \multicolumn{8}{|l|}{ Swath fields } \\
\hline IB6V01070 & 01 & $2010-02-13$ & F110W & $00: 22: 35.86$ & $-72: 10: 00.5$ & 3075.64 & 316.006 \\
\hline IB6V01080 & 01 & $2010-02-13$ & F160W & $00: 22: 35.87$ & $-72: 10: 00.5$ & 5993.86 & 316.006 \\
\hline IB6V02070 & 02 & 2010-03-04 & F110W & $00: 22: 09.51$ & $-72: 09: 34.5$ & 3075.64 & 336.113 \\
\hline IB6V02080 & 02 & 2010-03-04 & F160W & $00: 22: 08.42$ & $-72: 09: 32.8$ & 5993.86 & 336.117 \\
\hline IB6V03070 & 03 & 2010-03-16 & F110W & $00: 21: 48.75$ & $-72: 08: 36.7$ & 3035.64 & 354.198 \\
\hline IB6V03080 & 03 & $2010-03-16$ & F160W & $00: 21: 48.75$ & $-72: 08: 36.7$ & 5943.86 & 354.198 \\
\hline IB6V04070 & 04 & $2010-04-10$ & F110W & $00: 21: 29.65$ & $-72: 06: 44.9$ & 3075.64 & 17.276 \\
\hline IB6V04080 & 04 & 2010-04-10 & F160W & $00: 21: 29.65$ & $-72: 06: 44.9$ & 5993.86 & 17.276 \\
\hline IB6V25070 & 25 & 2010-05-03 & F110W & $00: 21: 21.81$ & $-72: 04: 40.1$ & 3075.64 & 38.310 \\
\hline IB6V25080 & 25 & $2010-05-03$ & F160W & $00: 21: 21.81$ & $-72: 04: 40.1$ & 5993.86 & 38.310 \\
\hline IB6V06070 & 06 & 2010-06-12 & F110W & $00: 21: 23.84$ & $-72: 02: 36.6$ & 2972.70 & 58.303 \\
\hline IB6V06080 & 06 & 2010-06-12 & F160W & $00: 21: 23.84$ & $-72: 02: 36.6$ & 5693.86 & 58.303 \\
\hline IB6V07070 & 07 & 2010-06-18 & F110W & $00: 21: 39.75$ & $-72: 00: 14.0$ & 3075.64 & 84.241 \\
\hline IB6V07080 & 07 & $2010-06-18$ & F160W & $00: 21: 39.75$ & $-72: 00: 14.0$ & 5993.86 & 84.241 \\
\hline IB6V08070 & 08 & $2010-07-29$ & F110W & $00: 21: 57.98$ & $-71: 59: 01.2$ & 2871.93 & 102.168 \\
\hline IB6V08090 & 08 & 2010-07-29 & F160W & $00: 21: 57.98$ & $-71: 59: 01.2$ & 5668.86 & 102.168 \\
\hline IB6V09070 & 09 & 2010-08-05 & F110W & $00: 22: 20.14$ & $-71: 58: 17.9$ & 3075.64 & 120.079 \\
\hline IB6V09080 & 09 & 2010-08-05 & F160W & $00: 22: 20.14$ & $-71: 58: 17.9$ & 5993.86 & 120.079 \\
\hline IB6V10070 & 10 & $2010-08-14$ & F110W & $00: 22: 46.75$ & $-71: 58: 09.5$ & 3075.64 & 139.972 \\
\hline IB6V10080 & 10 & 2010-08-14 & F160W & $00: 22: 46.75$ & $-71: 58: 09.5$ & 5993.86 & 139.972 \\
\hline IB6V11070 & 11 & 2010-09-19 & F110W & $00: 23: 11.19$ & $-71: 58: 40.9$ & 3075.64 & 158.873 \\
\hline IB6V11080 & 11 & 2010-09-19 & F160W & $00: 23: 11.19$ & $-71: 58: 40.9$ & 5993.86 & 158.873 \\
\hline IB6V12070 & 12 & 2010-10-01 & F110W & $00: 23: 32.14$ & $-71: 59: 47.4$ & 3075.64 & 177.788 \\
\hline IB6V12080 & 12 & 2010-10-01 & F160W & $00: 23: 32.14$ & $-71: 59: 47.4$ & 5993.86 & 177.788 \\
\hline \multicolumn{8}{|l|}{ Stare field } \\
\hline IB6V13070 & 13 & $2010-01-16$ & F110W & $00: 23: 12.24$ & $-72: 09: 25.4$ & 3004.95 & 287.360 \\
\hline IB6V13080 & 13 & 2010-01-16 & F160W & $00: 23: 12.24$ & $-72: 09: 25.4$ & 5743.87 & 287.360 \\
\hline IB6V14040 & 14 & $2010-01-17$ & F110W & $00: 23: 11.22$ & $-72: 09: 28.3$ & 4404.19 & 287.364 \\
\hline IB6V14030 & 14 & $2010-01-17$ & F160W & $00: 23: 10.23$ & $-72: 09: 30.9$ & 9941.56 & 287.368 \\
\hline IB6V15030 & 15 & 2010-01-18 & F110W & $00: 23: 10.22$ & $-72: 09: 31.2$ & 5785.59 & 287.368 \\
\hline IB6V15050 & 15 & 2010-01-18 & F160W & $00: 23: 12.20$ & $-72: 09: 25.9$ & 8748.82 & 287.360 \\
\hline IB6V16040 & 16 & 2010-01-19 & F110W & $00: 23: 11.79$ & $-72: 09: 27.1$ & 4378.42 & 287.680 \\
\hline IB6V16030 & 16 & 2010-01-19 & F160W & $00: 23: 09.80$ & $-72: 09: 32.3$ & 9967.33 & 287.688 \\
\hline IB6V17040 & 17 & $2010-01-20$ & F110W & $00: 23: 10.40$ & $-72: 09: 29.1$ & 4378.42 & 288.821 \\
\hline IB6V17030 & 17 & $2010-01-20$ & F160W & $00: 23: 08.39$ & $-72: 09: 34.2$ & 9967.33 & 288.829 \\
\hline IB6V18030 & 18 & $2010-01-20$ & F110W & $00: 23: 07.03$ & $-72: 09: 37.0$ & 5596.93 & 289.892 \\
\hline IB6V18040 & 18 & $2010-01-21$ & F160W & $00: 23: 09.07$ & $-72: 09: 32.1$ & 8748.82 & 289.884 \\
\hline IB6V19040 & 19 & $2010-01-25$ & F110W & $00: 23: 02.74$ & $-72: 09: 42.5$ & 4378.42 & 294.698 \\
\hline IB6V19030 & 19 & $2010-01-25$ & F160W & $00: 23: 00.62$ & $-72: 09: 46.6$ & 9967.33 & 294.706 \\
\hline IB6V20040 & 20 & $2010-01-23$ & F110W & $00: 23: 11.52$ & $-72: 09: 27.5$ & 4378.42 & 287.863 \\
\hline IB6V20030 & 20 & $2010-01-23$ & F160W & $00: 23: 09.52$ & $-72: 09: 32.7$ & 9967.33 & 287.871 \\
\hline IB6V21040 & 21 & 2010-01-26 & F110W & 00:23:01.62 & $-72: 09: 44.0$ & 4503.42 & 295.693 \\
\hline IB6V21030 & 21 & $2010-01-26$ & F160W & $00: 22: 59.49$ & $-72: 09: 47.9$ & 9842.33 & 295.702 \\
\hline IB6V22050 & 22 & $2010-01-27$ & F110W & $00: 23: 00.11$ & $-72: 09: 46.3$ & 4271.16 & 296.843 \\
\hline IB6V22030 & 22 & $2010-01-27$ & F160W & $00: 22: 57.96$ & $-72: 09: 50.0$ & 10074.6 & 296.852 \\
\hline IB6V23040 & 23 & $2010-01-28$ & F110W & $00: 22: 58.59$ & $-72: 09: 48.5$ & 4471.16 & 297.993 \\
\hline IB6V23030 & 23 & $2010-01-28$ & F160W & $00: 22: 56.42$ & $-72: 09: 52.0$ & 9842.33 & 298.002 \\
\hline IB6V24040 & 24 & $2010-01-15$ & F110W & $00: 23: 10.25$ & $-72: 09: 31.6$ & 5670.40 & 287.368 \\
\hline IB6V24030 & 24 & 2010-01-15 & F160W & $00: 23: 10.25$ & $-72: 09: 31.6$ & 11484.8 & 287.368 \\
\hline
\end{tabular}

${ }^{a}$ The observational $\log$ is grouped into $H S T$ visits, where each visit with WFC3/IR consists of three orbits in the Swath field (one for $F 110 W$ and two for $F 160 W$ ) and about five orbits in the Stare field for both filters. Multiple deep and shallow exposures are obtained in each orbit. For the Swath field, these are the same visits as those shown in Table 2 for the WFC3/UVIS observations.

${ }^{b}$ The total integration time for all deep and short observations.

we obtained with ACS and WFC3/IR was drizzled on to that grid (i.e., the $H S T$ images were also rescaled to 0.10 arcsec). The intensity of this master HST montage was scaled to match the ground-based image, the two were added together, and we retained pixels from the $H S T$ image only where they were available and those of the ground-based image otherwise.

The montage shows the primary ACS field from our study just west of the cluster center as a star-like pattern. This results from the multiple roll angles, each at $\delta \sim 20$ degrees. Surrounding this image in an arc are the multiple fields observed with WFC3, beginning at the bottom just south-east of the ACS field (the Stare field) and continuing to the north in the 12 separate Swath fields. This arc is illustrated here with the WFC3/IR imaging fields, whereas, the WFC3/UVIS coverage is much better.

A summary of all of the observations collected in this program is presented in Table 1 (ACS), Table 2 (WFC3/UVIS), and Table 3 (WFC3/IR). In these tables, all exposures that are obtained within single visits are grouped together, where the visits include mul- 
tiple dithered exposures over several orbits. The identifier in column 1 represents the association name for the first exposure in each group. More detailed information on the 707 individual exposures can be obtained from the Multimission Archive at STScI (MAST - http://archive.stsci.edu).

\section{IMAGE ANALYSIS AND STACKING}

The first images of this program were collected by $H S T$ in January 2010, just 4 months after STScI finished the Servicing Mission Orbital Verification (SMOV) program that provided a preliminary calibration of the repaired ACS and newly installed WFC3 instruments. For ACS, post SM4 calibration has shown that the instrument's behavior, including photometric throughput and anomalies, is similar to the existing baseline that was established before it shut down in January 2007. For WFC3, however, the learning curve has initially been very steep. Calibration reference files were produced with the first on-orbit data and these replaced the ground-calibration files. New on-orbit files have subsequently been released through the web pages and updated into the data reduction pipeline system as new knowledge of the respective instrument performance and defects has become available.

Given the frequency of these updates for WFC3, the data from the two instruments in this program were treated differently. For ACS, the calibrated _flt files which were produced using the calacs pipeline with postSM4 reference files were retrieved from MAST. Recent ACS images exhibit clear signatures of CTE degradation. Traps in the silicon lattice cause some charge to be delayed during readout, essentially blurring the profiles of sources away from the readout amplifier. Charge from a source is often delayed so much that it is recorded well outside of the aperture used to measure the object, with the result being that the object appears fainter than it truly is (see Figure 51). Not correcting for this effect would lead to both larger photometric and astrometric errors in the data, especially on short exposures with low sky background (Anderson \& Bedin 2010; Massev 2010). All ACS pipeline fft images were therefore subjected to the pixel based CTE correction task PixCteCorr in Python, which is described in detail on the STScI ACS webpages $^{11}$ and Anderson \& Bedin (2010).

For WFC3, the _raw images from the instrument were manually reprocessed with the latest calibration reference files that have been released as alpha and beta releases on the WFC3 web pages, often not yet ingested into the automated pipeline. The primary steps involved in both the automated and manual processing of _raw data from the CCDs included a bias subtraction, dark subtraction, flat fielding correction and gain conversion, as well as several other steps that populate the data quality and error arrays and calculate photometric header keywords. The WFC3/IR array is not a CCD and requires additional steps such as the "up-the-ramp" fitting to image units of count-rate. The manual processing of these new data with an updated version of the calwfc3 pipeline allows us to intervene at any stage and apply the latest reference files. Further details of the processing steps are provided in the WFC3 Data Handbooks,

\footnotetext{
11 http://www.stsci.edu/hst/acs/software/CTE/
}
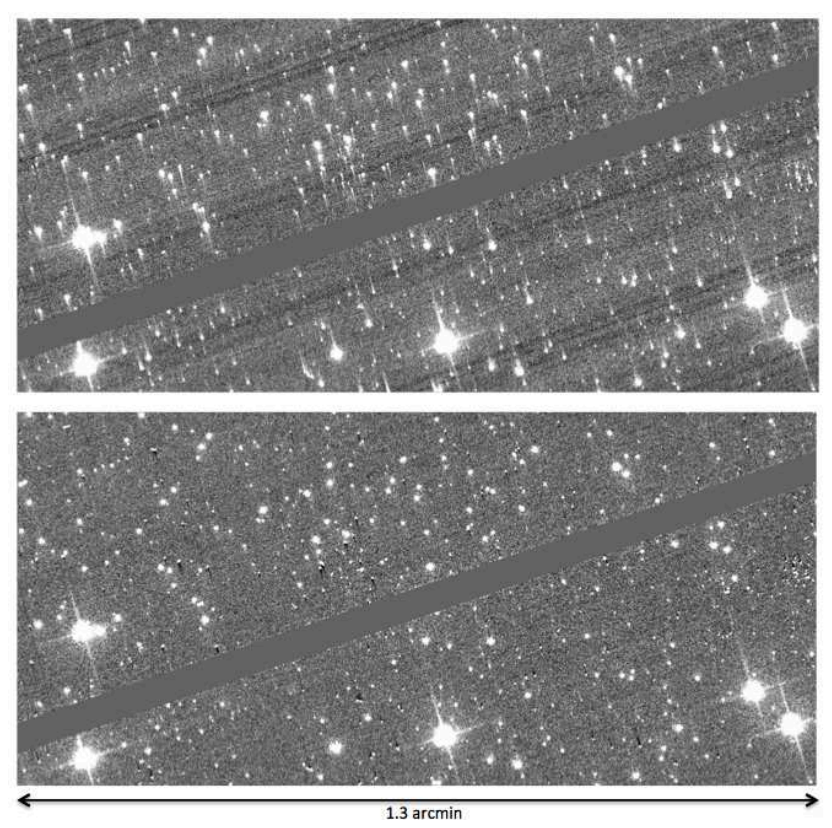

FIG. 5.- Top - An individual $10 \mathrm{~s}$ _flt image with a background of $\sim 1 e^{-}$from the ACS/WFC data shows significant effects of CTE losses. The trails are caused by electrons that were released after a short delay, being trapped due to pixel degradation of the detectors. The objects that are nearest to the chip gap shown are the furthest from the readout registers at the top and bottom of the detector, and therefore encounter the most charge traps and suffer the strongest trails. Bottom - The same image corrected for CTE losses using the methods of Anderson \& Bedin (2010). Both the flux and position of sources is restored. Note, the image in the top panel shows faint streaks that are parallel to the chip gap. These are present in post-SM4 ACS data, and are caused by readout electronics. Although the streaks look bad, their amplitude is very low. In the bottom panel, we remove these streaks using the acs_destripe Python task (Grogin et al. 2010).

\section{Rajan et al. (2010).}

The primary goal of this study is to find and measure the faintest possible stars in the imaging fields. These stars often represent $0.2 \sigma$ events relative to the background in individual images, and can only be seen by carefully analyzing all the of images together. There are two ways to do this. One, described in Anderson \& King (2000) and Anderson et al. (2008a; 2008b) involves examining all of the images together to find the places in the field where a statistically significant number of images registered a marginal detection. The pixels in all of the images in the vicinity of this location are then fit simultaneously to yield the most probable flux for the source, with careful attention to the PSF and $\mathrm{S} / \mathrm{N}$ in each pixel (e.g., Lauer 1999a). The final photometric measurements from this treatment of _flt images has led to exquisite CMDs for the bulk of the Milky Way globular clusters (e.g., Anderson et al. 2008a; 2008b), and the discovery of multiple stellar populations in these systems (e.g., Piotto et al. 2007 and Piotto 2009 and references therein).

An alternate approach for finding these faintest sources involves combining the multiple dithered _flt images for a given filter into a stacked image, and to perform photometric and astrometric measurements on the single coadded frame. Several techniques exist for achieving the stacked image, from interlacing of input pixels from 
multiple individual images onto a finer grid of different output pixels (e.g., Lauer 1999b) to linearly shifting and adding pixels on to the subsampled grid. Although interlacing can provide optimum combination of the images, the approach is limited in practice by several effects related to pointing accuracy and geometric distortion, as discussed in Fruchter \& Sosev (2009), Koekemoer et al. (2002), and references therein. An improved method of image combination specifically for undersampled $H S T$ data is called MultiDrizzle (Fruchter \& Hook 2002). In this algorithm, the individual pixels from the flt images (the input pixels) are rained down (or "drizzled") onto a common reference frame, which is often super-sampled relative to the input frames. To do this, the location of each pixel is first corrected for geometric distortion and then the entire frame of pixels is shifted, rotated, and stretched so that each pixel is mapped into its proper place in the master frame. The size of each input pixel is then shrunk to a smaller drop size than its original footprint (as described in Fruchter \& Sosey 2009). Although the MultiDrizzle approach still smears the final output image with the convolution of the drop size and the output pixels, in cases where the input images are well dithered, it is able to provide improved sampling of the PSF relative to the individual input images. This is especially important on wide-field HST cameras such as ACS, WFC3/UVIS, and WFC3/IR where the size of a native pixel is comparable to the full width at half maximum (FWHM) of the PSF.

To demonstrate that the scientific results are independent of analysis methods, we will analyze these data using both approaches discussed above. In this paper, we describe the latter analysis using MultiDrizzle which reduces the 707 images collected to a single stacked, supersampled image in each of the handful of instrument, filter, and exposure time combinations (e.g., one for deep frames and one for shallow). In a future paper, Anderson et al. (2012, in prep.), we will describe the complementary reduction which involves less convolution and more optimal weighting, but requires carefully solving for the spatially and temporally varying PSF on each input image.

We next describe the specific methods used to stack the ACS, WFC3/UVIS, and WFC3/IR data.

\subsection{MultiDrizzling the ACS/WFC Primary Field}

The data processing was started by running a first pass of MultiDrizzle on the flt frames to generate individual output versions of all $117 F 606 \mathrm{~W}$ and $125 \mathrm{~F} 814$ frames which have been corrected for the geometric distortion of the camera. ${ }^{12}$ The distortion solution comes from the ACS calibration IDCTAB, u7n18502j_idc.fits. The accuracy of the WCS system and therefore the absolute alignment of these drizzled ("single_sci") images is limited by an intrinsic accuracy of $\sim 0.5$ arcsec in the Guide Star Catalog. To improve the fine alignment, a transformation is iteratively calculated between each of the distortion-corrected drizzled frames by isolating several

\footnotetext{
12 Although the STScI calibration pipelines also produced_drz images, these are constructed with a default set of parameters for sky subtraction, rejection of bad pixels, and also do not include fine adjustments in image registration. The overall quality of these combined images is much worse than what can be achieved by manually reprocessing the data.
}

hundred bright stars on each image and measuring their centroids using a Gaussian profile. The transformation between these catalogs allows for linear offsets, rotations, and scale changes to refine the estimates of the Guide Star Catalog positions, account for the residual roll angle of the telescope from what was asked for, and factor in any breathing changes. The solution was refined through successive matching of the common stars down to a tolerance of 0.3 pixels. With the large number of stars available in the cluster, the final offsets provide an alignment of the individual images that is better than 0.01 pixel.

These new offsets, rotations, and scales were supplied to MultiDrizzle in a second pass as a "shift" file and added to the WCS header information. In this new stack of images, only real astrophysical sources are aligned, thereby providing a robust means to identify pixels that are affected by cosmic rays or are otherwise problematic (e.g., bad or hot pixels). The different bits that had already been set in the _flt images based on data quality arrays and bad pixel tables were largely ignored since the data provides a sensitive screening of those pixels that are specifically affecting the quality of our images. For example, the multiple roll angles of the observations provide a means to largely eliminate diffraction spikes from bright stars, which march around their respective stars through the aligned sequence across different visits. In addition to tweaking the location of each exposure with respect to the reference frame, offsets in the sky background of each exposure are accounted for and adjusted to match more closely that of the stack. This provided a much better rejection of discordant pixel values. Unlike extragalactic deep fields, the background of the images in this program is affected by the bright stars and so the purpose here is to ensure that any systematic offset level is accounted for prior to image combination. The background on each image was calculated as the modal sky value after clipping pixels that are more than $5 \sigma$ deviating in multiple iterations. A new bad-pixel mask was constructed by first producing a sigma-clipped median image in each of the two filters, and then reverse-drizzling (or "blotting") the median image back to the respective dither and roll angle of every original input image in the stack. These blotted images were next compared to the input _flt distorted images to statistically reject all bad pixels and cosmic rays. This produces both clean versions of the input data as well as output masks with the bad pixels.

The final step of creating the image stack involved a third pass of MultiDrizzle to combine all of the input undistorted and aligned frames into a final image, utilizing the updated mask files to screen out bad pixels and cosmic rays. ${ }^{13}$ At this stage, we took further advantage of the multiple dither and roll angles to improve sampling of the PSF and to increase the spatial resolution of the final output image. For ACS, the FWHM of the PSF in the flt images is $1.7-1.8$ pixels in $F 606 W$ and $F 814 W$ (i.e., at the native resolution of $0.05 \mathrm{arcsec} / \mathrm{pixel}$ ). The pixels in all of the input images are shrunk and "dropped" down onto an output supersampled image with a scale of $0.03 \mathrm{arcsec} /$ pixel. For this process, we used a circular

13 We verified that drizzling yielded the expected gains in image depth by performing this step on smaller subsets of the data before executing the full stack. 


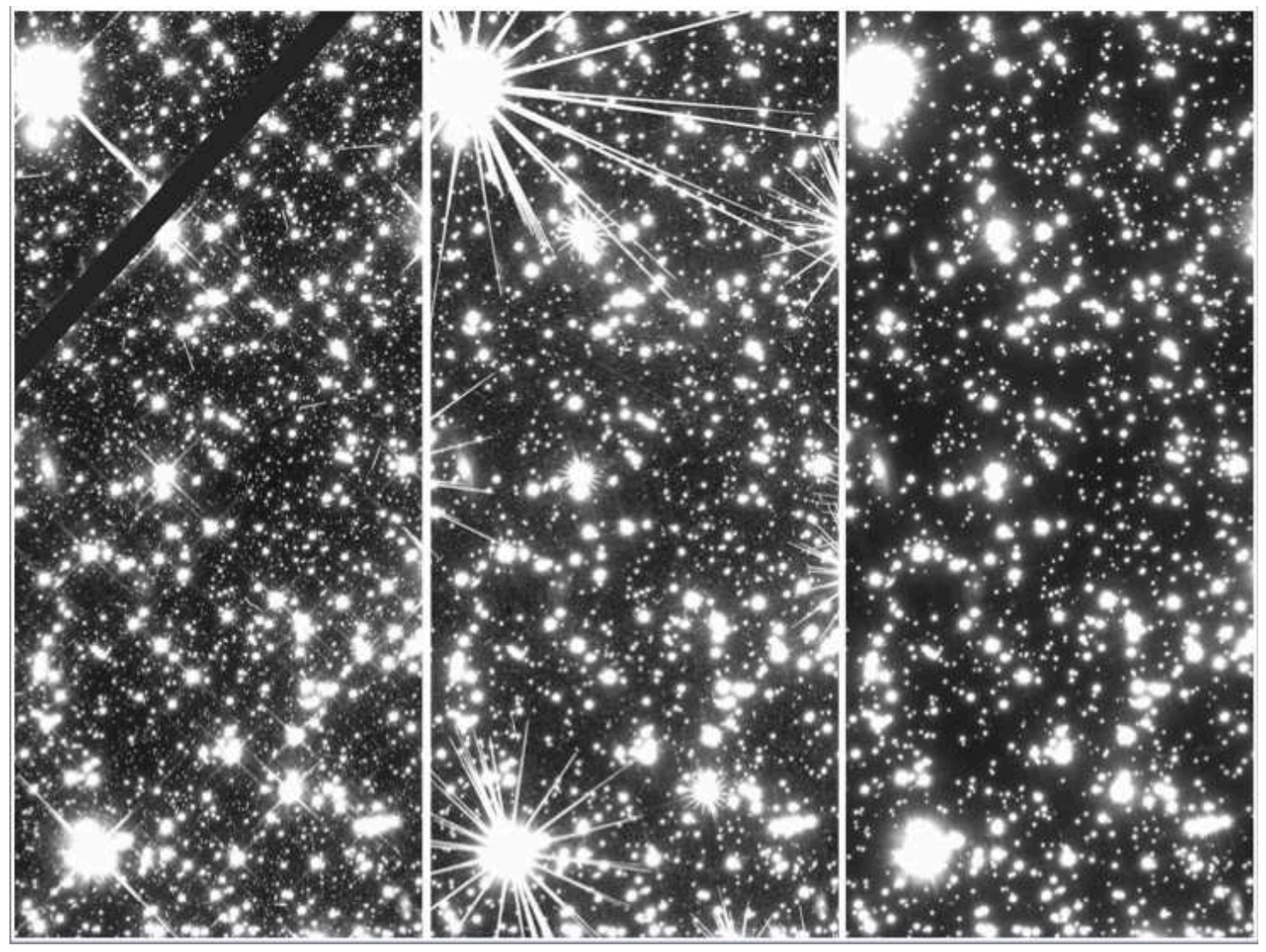

FIG. 6. - The quality of the final drizzled image for the ACS primary field is shown (right), relative to a single image that went into the stack (left) and a summed image of all 125 input images (middle). The single image exhibits many artifacts caused by bad pixels and cosmic rays, as well as the obvious chip gap separating the two CCDs on the ACS/WFC. The most noticeable feature in the summed image is the large number of diffraction spikes surrounding the bright stars, each set corresponding to a unique roll angle of the observations. Many of the cosmic rays were removed in generating this image to prevent it from being saturated with artifacts. The final drizzled image in the right panel recovers information that is lost in the gap between the chips and is free of almost all artifacts. The image shown here was generated with a Gaussian kernel, although we also experimented with kernels that gave a sharper final output image. The one set of barely perceptible diffraction spikes is caused by the large number of exposures at approximately fixed roll angle for the Stare-field observations. Each of the panels in this image extends about 40 arcsec in the horizontal direction.

Gaussian kernel with "pixfrac" $=0.7$ to distribute the flux onto the output grid. This process conserves flux by computing the overlapping area between each of the input drops and output pixels, and dividing the flux that is drizzled down by appropriate weights. The final combined image exhibits a well sampled PSF with $\sim 2.7$ pixel FWHM. The image spans $\sim 10,500$ pixels in both directions.

In generating the final drizzled images, we also experimented with several different kernel functions for combination and choices of scale and pixfrac. The Gaussian kernel leads to a slight "blurring" of the stars in the image relative to a square kernel (e.g., see Figure 6), but we found that our PSF modeling was slightly better in this case. The balance between scale and pixfrac needs to ensure that the output image is not convolved with too large of an input-pixel drop size, yet that the drop size is large enough to provide output pixels that have data in them from a sufficient number of the input images (especially towards the edges of the frame). The fidelity of the combined image was evaluated by examining a map of the relative weights of the output pixels. If the pixels had been shrunk too much, large populations of output pixels would have little or no contribution from the in- put pixels, and this would lead to an increased standard deviation in the weight image. If such large variation existed, photometric precision of astrophysical sources would be compromised. According to Fruchter \& Sosey (2009), the ratio between the root-mean-square and median of this image (away from the edges) should be $\lesssim 20$ $-30 \%$. This constraint is comfortably satisfied at all locations in the final ACS drizzled image given the large number of input images.

A zoomed in view of a small portion of the final drizzled ACS field is presented in the right panel of Figure 6 . This image is for the $F 814 W$ filter and spans $\sim 40$ arcsec in the horizontal direction. The image is mostly free of artifacts and highlights a very clean view of the stellar populations, and also reveals a number of face-on and edge-on spiral and elliptical galaxies and some interacting pairs. The one set of faint diffraction spikes around bright stars is caused by the approximately fixed orientation for half of the orbits (i.e., the roll angle for the Stare field). For comparison, a single frame in the drizzled stack is shown in the left panel and shows many artifacts from bad pixels and cosmic rays, as well as the chip gap between the two CCDs. A summed image of all of the aligned single frames is shown in the middle panel 
and shows diffraction spikes corresponding to each of the roll angles in the observations. Many of the cosmic rays in this summed image were clipped for display purposes (i.e., to prevent it from being saturated with artifacts).

In the ACS/WFC deep exposures, stars with $\sim 19$ th magnitude were saturated on the detector. The photometry and astrometry of these stars is therefore compromised. To ensure that a complete stellar census is measured including bright giants in 47 Tuc, the observations that were obtained with shorter integration times were also reduced independently (discussed in Section 2.1). The same methodology to that described above was adopted for these, but, given the more limited sampling information and resulting weight image statistics, the pixfrac was left at unity in the final combination. This process produced a single stacked image in each of the two filters, for each of the $100 \mathrm{~s}, 10 \mathrm{~s}$, and $1 \mathrm{~s}$ short exposures.

\subsection{MultiDrizzling the WFC3/UVIS and IR Parallel Fields}

The WFC3/IR array offers many advantages over conventional CCDs that work to our advantage, especially in cases where only a few images were obtained of a given field (i.e., all observations in this program except for the IR Stare field - see Section 2.2). Accumulated charge is electronically read out from the camera during an exposure through multiple non-destructive reads. Artifacts such as cosmic rays can therefore easily be identified and removed by fitting the count rate from these multiple reads (i.e., pixels affected by cosmic rays stood out in the read in which the hit impacted the array).

All of the _raw WFC3/UVIS and IR data were processed into calibrated flt frames using the latest reference files available on the WFC3 webpage (i.e., the new alpha-release flat fields). The _flt files were input into MultiDrizzle and processed using the same prescription described above for ACS (i.e., using the uab1537ci_idc.fits IDCTAB). This ensured an accurate alignment of all exposures in a given filter, sky subtraction, and the masking out of deviant pixels (using the "minmed" option instead of the median). The final image stack for WFC3/UVIS was generated at the native resolution of $0.04 \mathrm{arcsec} / \mathrm{pixel}$, whereas the $\mathrm{WFC3/IR}$ images were slightly supersampled to a pixel scale of $0.09 \mathrm{arcsec} / \mathrm{pixel}$ to mitigate the severe undersampling of these data (native FWHM $=1.1-1.2$ pixels). A square kernel was used in the final image generation and the pixfrac was kept near unity, guided by an iterative process of performing photometry on the resulting stacked image and choosing the input set up that leads to the best defined CMDs (see below).

For the IR Stare field, the observations contained $38 F 110 W$ and $80 F 160 W$ deep exposures with many dithers and a small amount of roll. The area on the sky mapped out by WFC3/IR during these 59 orbits is about $60 \%$ larger than a single WFC3/IR field, as shown in an effective exposure time map (i.e., a "weight" image) in Figure 7. These data are more similar to the primary-field observations and can be combined to produce a higher-quality drizzled image than in the Swath. The same techniques as described above were again followed to align and clean all of the input images using MultiDrizzle, and to combine them into the final stack.
The drizzled image was supersampled from the native resolution of $0.13 \mathrm{arcsec} / \mathrm{pixel}$ to $0.06 \mathrm{arcsec} / \mathrm{pixel}$, with a Gaussian kernel with pixfrac $=0.70$. The FWHM on this image is $\sim 2.5$ pixels. As with the ACS primary field, the weight image of the Stare field was inspected to verify that the standard deviation in the output pixels was well below the median (see Figure 7). An example of the final drizzled image for both the Swath and Stare fields is shown in Figure 2 .

In addition to the deep observations, multiple short exposures were obtained on both WFC3 cameras to mitigate saturation (see Section 2.2). For WFC3/UVIS, the short exposures consisted of two $50 \mathrm{~s}$ observations in each filter. These were drizzled together as described above. For WFC3/IR the shorter exposures were analyzed, but they did not add any new information on top of what was recovered in the deep frames. The reason for this is again related to the "up-the-ramp" fitting of the signal on the IR array through multiple non-destructive reads, the shortest of which have integration times of just a few seconds in which even the brightest stars do not saturate. This is described more in Section 4.3 .

\section{PHOTOMETRY}

The image-processing steps described in the last section combined hundreds of individual exposures into a handful of drizzled images. For the primary ACS field, we now have a single $F 606 W$ and $F 814 W$ well-sampled deep exposure and corresponding shallower images. For the Swath and Stare fields, a montage of WFC3/UVIS (deep and shallow) and WFC3/IR (deep) exposures over 13 different fields have been produced and also drizzled together as shown in Figure 2. The observations in the IR Stare field are well sampled, but the Swath is undersampled.

To measure the photometry, astrometry, and morphology of all sources, the standalone versions of the DAOPHOT II and ALLSTAR photometry programs were used (Stetson 1987, 1994). These programs offer flexibility in modeling the PSF with a range of analytic functions and account for some of its spatial variations across the frame (Stetson 1992). These variations are known to be significant for HST cameras such as ACS/WFC (Anderson et al. 2008a), and are currently being characterized for WFC3. Such PSF-fitting methods can improve the signal-to-noise of measurements over aperture photometry and also provide a much better characterization of stars that are either located close to other stars and have contamination from the neighbor and/or blended stars that directly overlap one another.

\subsection{PSF Photometry in the ACS/WFC Primary Field}

To analyze the well-sampled ACS/WFC drizzled images in each filter, a first pass of DAOPHOT was performed to yield positions of all possible detections in both deep images. These were taken to be sources that are at least $2.5 \sigma$ above the local sky. Photometry of these candidates was estimated in each filter independently, using an aperture with $R=2$ pixels. The images in each filter were treated independently at every stage of the analysis, and therefore there is a transformation that connects them. This transformation was derived by first selecting several hundred bright stars and crosscorrelating the lists to define an $x$ and $y$ offset, rotation, 


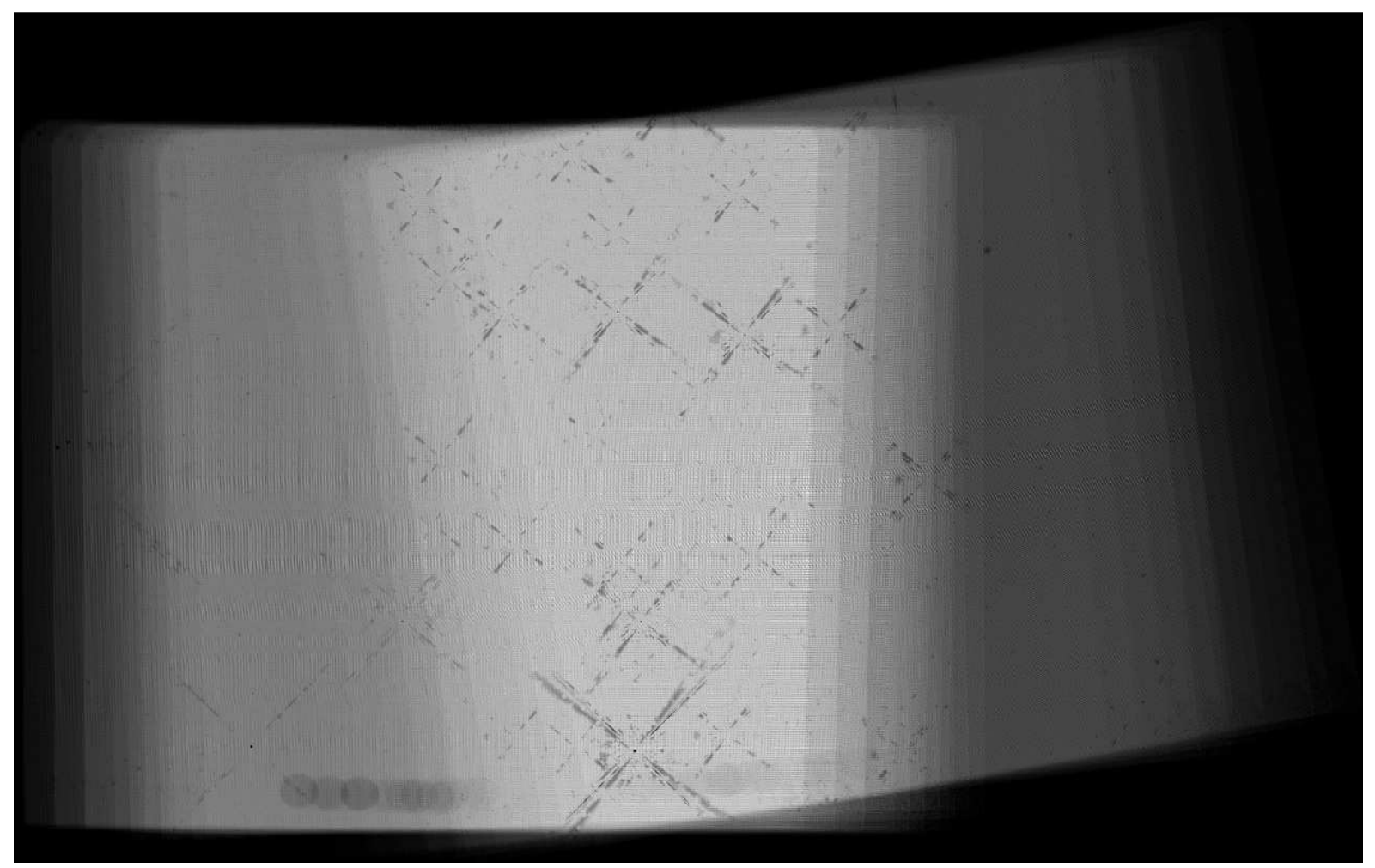

FIG. 7.- The weight image of the final combined WFC3/IR Stare field in $F 160 W$. The shading indicates an effective exposure time map, where regions near the center of the image have 80 overlapping independent images. The "slosh" around the center is caused by small roll changes in the primary ACS observations over 59 orbits of the program. These \pm 5 degree roll changes result primarily from scheduling constraints and work to provide wider field coverage in the parallel observations. Several artifacts can be seen in this image, such as diffraction spikes from bright stars (note, there is no bleeding of saturated pixels in the IR array) and the "death star" on WFC3/IR in the lower left - a region of dead pixels. As for the ACS primary field, this weight image was inspected to verify that the final choices of output pixel scale and drop size in MultiDrizzle were appropriate given the input dither pattern.

and scale. Based on these initial values, the transformation was refined by feeding the cross correlation the full list of detections in each image and iterating the resulting solution by successively decreasing the matching radius tolerance down to 1.0 pixel. These two lists were combined and a first CMD was generated consisting of $>70,000$ measurements.

Next, 1000 PSF candidate stars were selected from this merged list in each filter. These stars were required to be isolated (no neighbors within 19 pixels), not saturated, and to have a magnitude and color that placed them on the dominant stellar sequences in the CMD. The PSF of each image was calculated through an iterative method. Initially, all 1000 stars were used to build a spatially constant PSF, which was represented by the sum of an analytic function and an empirical look-up table representing the correction of the function to the observed brightness of the average profile of stars. For the analytic function, the sum of a Gaussian and a Lorentzian with five free parameters was used, as described in Stetson (1992). After fitting the analytic function to the observed stellar profiles of the PSF stars, the DAOPHOT-reported root-mean square residuals $\left(\chi^{2}\right)$ of the brightnesses was inspected and PSF stars that showed scatter more than twice the average were eliminated from the list. This entire process was repeated a second time with a PSF that was allowed to vary linearly with position in the frame, a third time allowing for quadratic variations, and a fourth time allowing for third order polynomial variations (i.e., cubic). The number of initial PSF candidates that passed these successive iterations was 881 for $F 606 W$ and 874 for $F 814 W$, and the final $\chi^{2}$ is $\lesssim 0.02$ indicating that the analytical function fit the observed profiles to within about $2 \%$. This residual, a systematic difference, formed the look-up table of profile corrections.

The final step of the processing applied the PSF defined above to the catalog of all sources in each image. For this, ALLSTAR was used to perform both PSF-fitted astrometry and photometry, and also to retain morphological information of sources through the SHARP diagnostic. SHARP provides an estimate of the concentration of the source with respect to the PSF, which gives us a way to discriminate between stars and galaxies.

The same procedure described above was repeated on each of the drizzled shallow exposures in the ACS primary field, yielding independent catalogs of the brighter stars.

\subsection{PSF and Aperture Photometry in the WFC3 Parallel Fields}

As discussed in Section 3.2, the WFC3 parallel Swath and Stare field observations have been reduced into a 

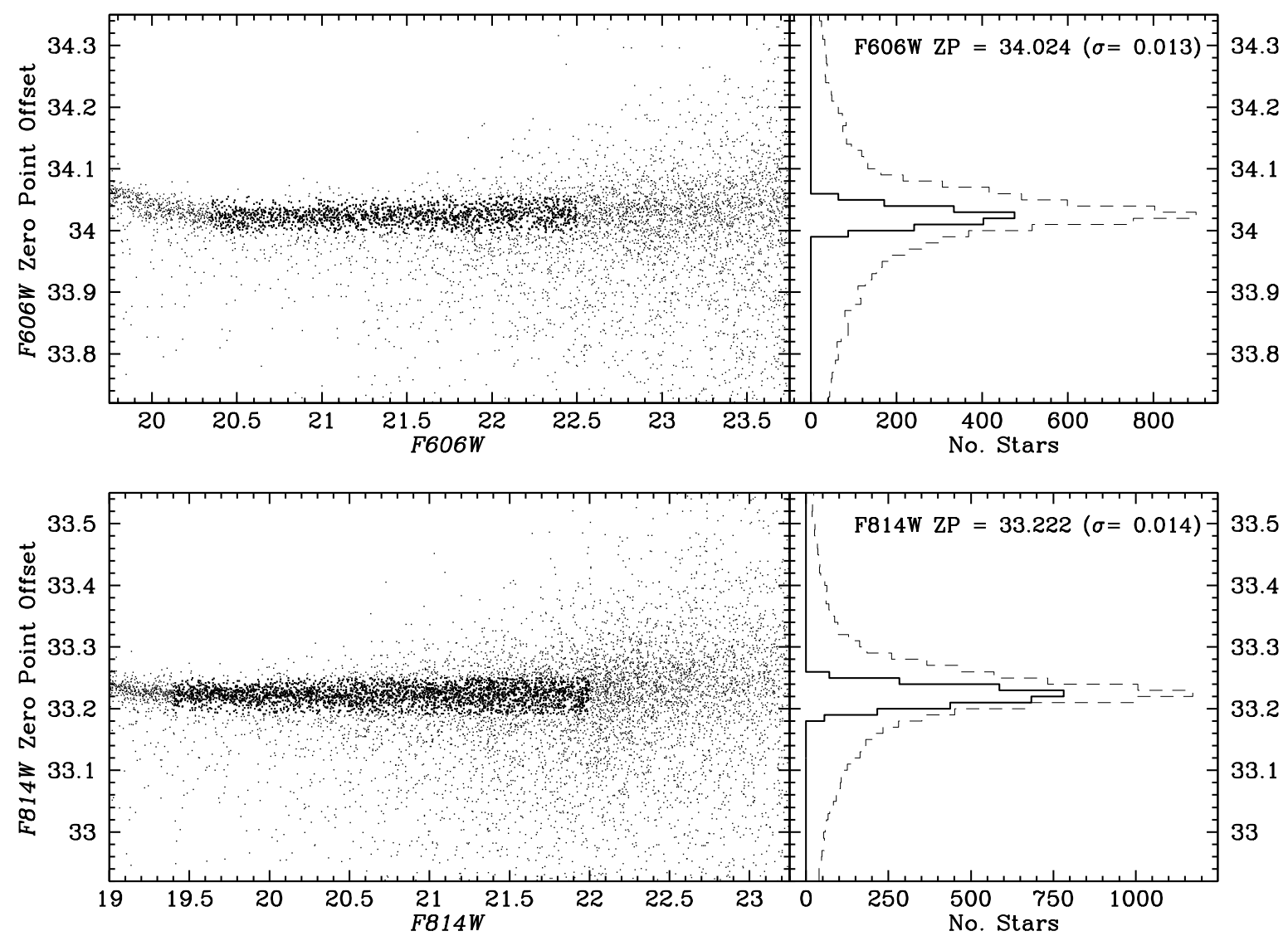

FIG. 8. - The derivation of the photometric zero points that are specific to the ACS primary field. The left panels show the difference between 5 pixel aperture photometry on a single drizzled image at the native scale and the PSF-fitted photometry on the super-sampled stacked image. The 5 pixel photometry has been calibrated to the VegaMag photometric system using the ACS zero points (Bohlin 2007). Stars with poor measurements due to faint neighbors were eliminated (e.g., the bias towards lower values in the plot) and zero points were calculated from the resulting distribution (darker points). These yield offsets of $F 606 W \mathrm{ZP}=34.024$ and $F 814 W \mathrm{ZP}=33.222$ for these reductions. Histograms of the distributions are shown in the panels on the right.

single drizzled image for each field and filter. The focus of the Swath fields is to sample a wide area of the cluster periphery, and therefore there are very few overlapping images in any given filter (see Section 2.2). The result is that we are unable to improve the sampling for these drizzled stacks. As this investigation is one of the first studies of crowded regime photometry with WFC3, different photometric methods were tested on these images to see which yields the most accurate photometry. This includes both aperture and PSF-fitted photometry on both the drizzled images and the _flt images (which required a correction for the pixel area map - Kalirai et al. 2010b).

For the slightly undersampled WFC3/UVIS Swath observations, PSF-fitted photometry on coadded images with DAOPHOT II and ALLSTAR produced sequences in the CMDs that extended fainter and were tighter than those produced with the other methods mentioned above. The prescription used for this was identical to the discussion above on the ACS primary field. The $\chi^{2}$ residuals from the analytic fit to the stellar profiles, after multiple iterations of the PSF, was measured to be $2-4 \%$ over the 13 fields. A similar procedure was also performed independently on the shallower drizzled exposures.

For WFC3/IR, the Swath data were too undersampled to accurately derive a PSF from the frame with DAOPHOT II. The photometry of all sources was there- fore measured using an aperture with $R=3.5$ pixels, where each pixel is 0.09 arcsec in the resampled images. Our independent analysis of the _flt images in the Swath fields for both cameras provide very similar photometric results to the drizzled images.

Unlike the WFC3/IR Swath fields, the Stare field was drizzled to an output scale of $0.06 \mathrm{arcsec} / \mathrm{pixel}$, and is therefore well sampled. For this field, a spatially varying PSF with DAOPHOT II was calculated as described above and was used with ALLSTAR to fit the stellar position, flux, and morphology of all sources.

\subsection{Merging the Bright and Faint Starlists}

The bulk of the imaging exposures in this program were obtained with integration times of $>1000 \mathrm{~s}$, and were intended to optimize the signal-to-noise of faint stars in 47 Tuc. The brightest cluster giants are more than 10 million times brighter than the faintest dwarfs, and therefore many stars saturated on both the ACS and WFC3/UVIS CCD observations. Accurate photometry for these bright stars was measured from the short exposures described earlier. The individual catalogs from each of the drizzled images with unique exposure times were "stitched" together by first finding well-measured, non-saturated, and isolated stars in each group and calculating a transformation between the coordinates of these stars. The photometry of the bright frames was 
zeropointed to the deep frame using these common stars, and all stars in the bright frames which were not measured in the deep frame were mapped into the catalog after applying the transformation. In this way the catalog contains uniform photometry and astrometry that is based on the deep drizzled image.

For the WFC3/IR frames, the multiple non-destructive reads provide accurate count-rates for most of the bright stars in the data set. The deep observations were constructed with the STEP200 and STEP400 sample sequences with at least 12 ramps, and these included reads at $2.9 \mathrm{~s}, 5.9 \mathrm{~s}, 8.8 \mathrm{~s}, \ldots 1399 \mathrm{~s}$ (see WFC3 Instrument Handbook - Dressel et al. 2010). Although the effective read noise is larger for sources that have count rates estimated from just a few reads, this is overwhelmed by the photon statistics from such bright stars. Therefore, with a single exposure time, we were able to measure stars spanning 13 magnitudes in the IR CMD. ${ }^{14}$

\subsection{Producing a Panchromatic Catalog}

Thus far, the analysis has treated the WFC3 data from the UVIS and IR cameras separately. The WFC3/UVIS field of view is $\sim 60 \%$ larger than the IR fields, and so the complete contiguous arc shown in Figure 2 (the IR fields) contains overlapping UVIS observations in $F 390 \mathrm{~W}$ and $F 606 \mathrm{~W}$. A transformation was next calculated to map the drizzled IR astrometry onto the UVIS reference frame, accounting for small rotations, shifts, and the large plate-scale change of the two cameras (the native UVIS pixels are $>2 \times$ smaller than even the supersampled IR pixels). The result of this transformation is a catalog with common stars, measured in any number of multiple filter combinations. A significant fraction of the stars have measurements in all four bandpasses, providing panchromatic imaging from $0.4-1.7$ microns (see next Section).

\subsection{Photometric Zero Points}

The photometric zero point of an instrument/filter combination is a convenient way to characterize the overall sensitivity. Conventionally, the zero point represents the magnitude of a star-like object that produces one count per second within a given aperture (see Maiz Apellaniz 2007). For HST instruments, these zero points have been defined for each instrument by observing spectrophotometric standard stars with well measured fundamental parameters (e.g., $T_{\text {eff }}$ and $\left.\log (g)\right)$ and STIS fluxes (Holtzman et al. 1995; Sirianni et al. 2005; Kalirai et al. 2009a; 2009b). The photometry of these isolated stars in large apertures is compared to the inferred sensitivity from the spectrum (or model) to provide the required calibration of the total system throughput of the filter (i.e., including the HST optics, detector quantum efficiency, filter transmission function, and many other components).

To place the 47 Tuc photometry on an absolute scale, the brightnesses of isolated stars on a single drizzled image are re-measured. This (single_sci) image has the geometric distortion removed but has not been otherwise altered (e.g., rescaled). Although the default photometric

\footnotetext{
14 The phase II program and APT design of these observations was submitted in December 2008, well before Servicing Mission 4 and any knowledge of instrument on-orbit photometric performance with limited numbers of reads.
}

TABLE 4

Photometric Zero Points for Final Reductions

\begin{tabular}{lcc}
\hline \hline \multicolumn{1}{c}{ Filter } & Zero Point & $\sigma$ \\
\hline ACS & 34.024 & 0.013 \\
$F 606 W$ & 33.222 & 0.014 \\
$F 814 W$ & & \\
& & \\
WFC3/UVIS & 32.851 & 0.028 \\
$F 390 W$ & 33.697 & 0.023 \\
$F 606 W$ & & \\
& & \\
WFC3/IR Swath Fields & 33.604 & 0.013 \\
$F 110 W$ & 32.197 & 0.011 \\
$F 160 W$ & & \\
& & \\
WFC3/IR Stare Fields & 33.673 & 0.015 \\
$F 110 W$ & 32.253 & 0.016 \\
$F 160 W$
\end{tabular}

zero points are typically given for an "infinite" aperture that sums the total flux of the star, this can be rescaled to a smaller aperture using the enclosed energy curve for the respective camera (see Sirianni et al. 2005 for ACS and Hartig 2009a; 2009b; Kalirai et al. 2009a; 2009b for WFC3/UVIS and IR). ${ }^{15}$ This rescaling ensures that the photometry has sufficient signal-to-noise and is not compromised by neighboring stars in the cluster.

An example of the zero-point derivation for the ACS/WFC primary field photometry is illustrated in Figure 8. For this, the photometry of stars in a single drizzled image at the native scale in each filter was measured using an aperture with radius 5 pixels $(0.25$ arcsec), and the infinite ACS VegaMAG zeropoint of 26.4060 in $F 606 \mathrm{~W}$ and 25.5199 in $F 814 W$ was added. ${ }^{16}$ Next, a correction of -0.151 and -0.171 was applied to translate the zero point to the aperture size based on the enclosed energy curve. The difference between this calibrated photometry and the final PSF-fitted photometry from Section 4.1 is shown on the vertical axis as the zero point specific to our measurements. The scatter in this diagram is driven by faint stars and cosmic rays that fall within the 5 pixel radius on the single image, so the distribution was $\sigma$ clipped to isolate the tight sequence of "clean" measurements (shown as larger points). Saturated stars at the bright end of the distribution, with $F 606 W \lesssim 20$ and $F 814 W \lesssim 19$, were also eliminated. The final zero point offsets, as measured from a few thousand stars, are $34.024(\sigma=0.013)$ in $F 606 W$ and 33.222 $(\sigma=0.014)$ in $F 814 W$ and have errors $<0.001 \mathrm{mag}$.

A similar analysis was performed on the WFC3/UVIS and IR Swath and Stare fields, using the photometric zero points published in Kalirai et al. (2009a) and Kalirai et al. (2009b) for an aperture of radius 10 pixels (UVIS) and 3 pixels (IR). The derived zero points for these reductions are summarized in Table 4.

\section{ARTIFICIAL-STAR TESTS}

\footnotetext{
15 The flux in an infinite aperture is calculated by first measuring photometry of the isolated standard out to several arcseconds (e.g., $\sim 100$ pixels on ACS/WFC and WFC3/UVIS) and then applying a small correction (typically $\lesssim 2 \%$ ) based on a model of the enclosed energy curve.

16 The magnitude of a star with flux $f$ in the VegaMAG system is simply $-2.5 \log \left(f / f_{\text {Vega }}\right)$, where $f_{\text {Vega }}$ is the calibrated spectrum of the star Vega.
} 

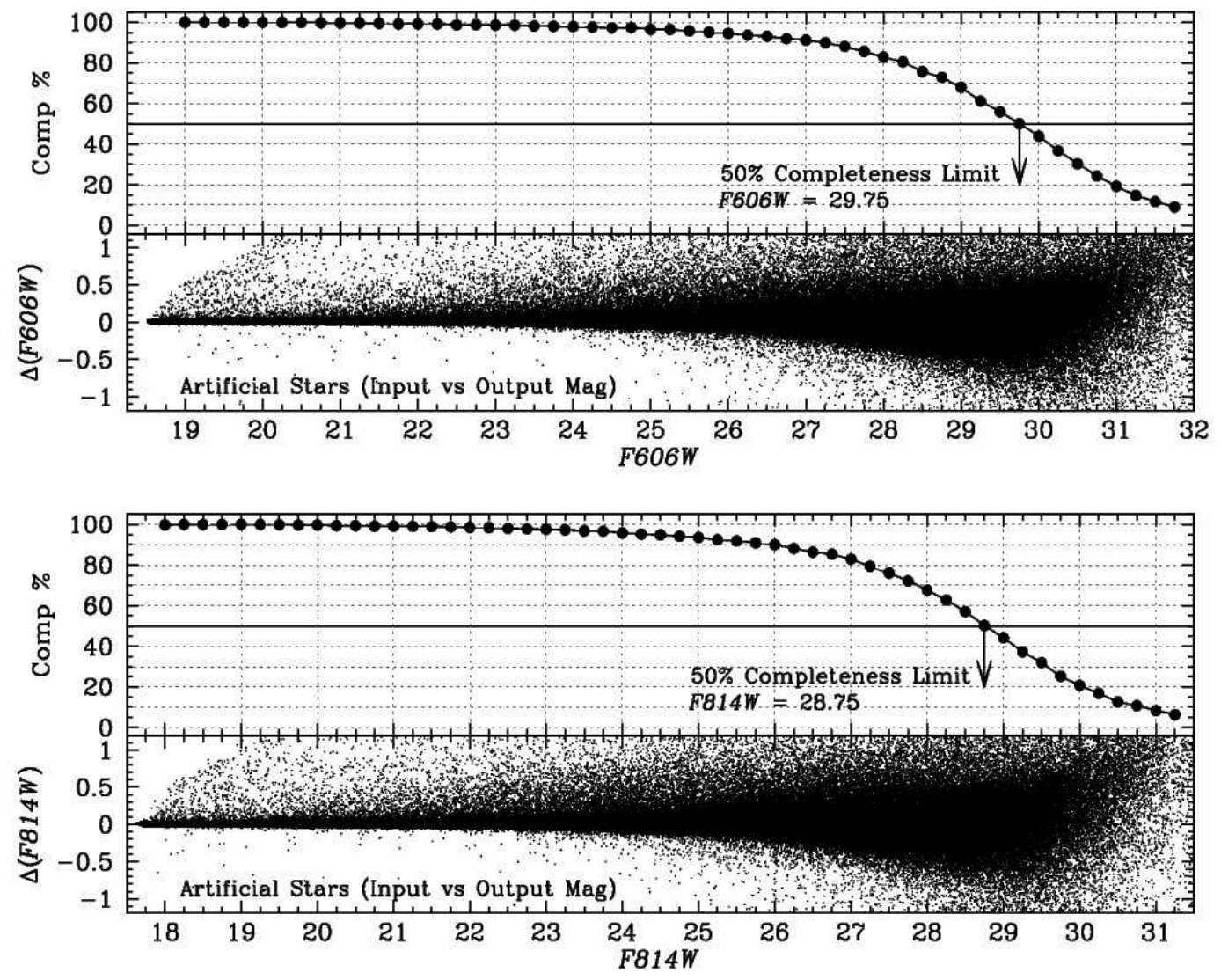

FIG. 9. - The completeness fraction of the ACS/WFC stacked images is measured through artificial-star tests. The top panels illustrate the completeness curve and photometric error distribution (e.g., input artificial star magnitude vs recovered magnitude) for the $F 606 W$ image and the bottom panels illustrate the same for the $F 814 W$ image. The $50 \%$ completeness limits are measured to be $F 606 W=29.75$ and $F 814 W=28.75$. Similar tests were also performed on each field of the Swath and Stare, in both the WFC3/UVIS and WFC3/IR data.

We characterize the photometric and astrometric error distribution of the final photometry in our images, as well as the completeness of the data reductions by performing an extensive set of artificial-star tests. These tests are tailored to each of the individual data products, including all six filters observed with ACS, WFC3/UVIS, and WFC3/IR, and are computed separately for each individual field to fully account for crowding variations due to the wide-field coverage of 47 Tuc.

The artificial stars are modeled from the PSF of each individual field, and scaled to reproduce the complete luminosity range of real stars in the deep drizzled stacks of each filter. The fraction of stars injected into each image was set to $\lesssim 5 \%$ of the total number of stars in the image, so as to not induce incompleteness due to crowding in the tests themselves. 500 trials were generated for each test combination (e.g., the observations in WFC3/UVIS field Swath 4, in the F390W filter) to form the input grid of artificial starlists and resulting images. These images were next subjected to the photometric routines that were applied to the actual drizzled images, using identical criteria. The stars were recovered blindly and automatically cross-matched to the input starlists containing actual positions and fluxes.

The artificial-star tests from this program can be used to investigate a number of questions regarding the fidelity of our reductions. For example, these tests are essential to the derivation of the stellar mass function of 47 Tuc.
We highlight one aspect of the tests in Figure 9 by presenting the incompleteness curve for our ACS primary field $F 606 W$ and $F 814 W$ observations, as well as the photometric error distribution (e.g., artificial star input vs output magnitude). These results indicate that the $50 \%$ completeness fraction of the ACS data is $F 606 \mathrm{~W}$ $=29.75$ and $F 814 W=28.75$. The completeness limits in the WFC3/UVIS and IR data vary by more than one magnitude depending on the field observed. Specific details will be presented along with the corresponding scientific investigations in future papers. As a reference point, in the Swath 3 field, the the $50 \%$ completeness is $F 390 W=28.3, F 606 W=28.8, F 110 W=26.2$, and $F 160 W=25.1$.

The total number of artificial stars generated in these tests exceeds 2 million and total 5 TB of disk space.

\section{COLOR MAGNITUDE DIAGRAMS}

CMDs from the PSF-fitted photometry of the final drizzled images in the UV, visible, and IR are presented in Figures 10 and [11, and a panchromatic CMD over the full wavelength from $0.4-1.7$ microns in Figure 12 . These CMDs provide a convenient summary of the observations in our 121 orbit program, and the quality of the data and data analysis. The primary features and depth of the CMDs is discussed here in relation to the program's science goals, which will be explored in greater detail in future papers. 


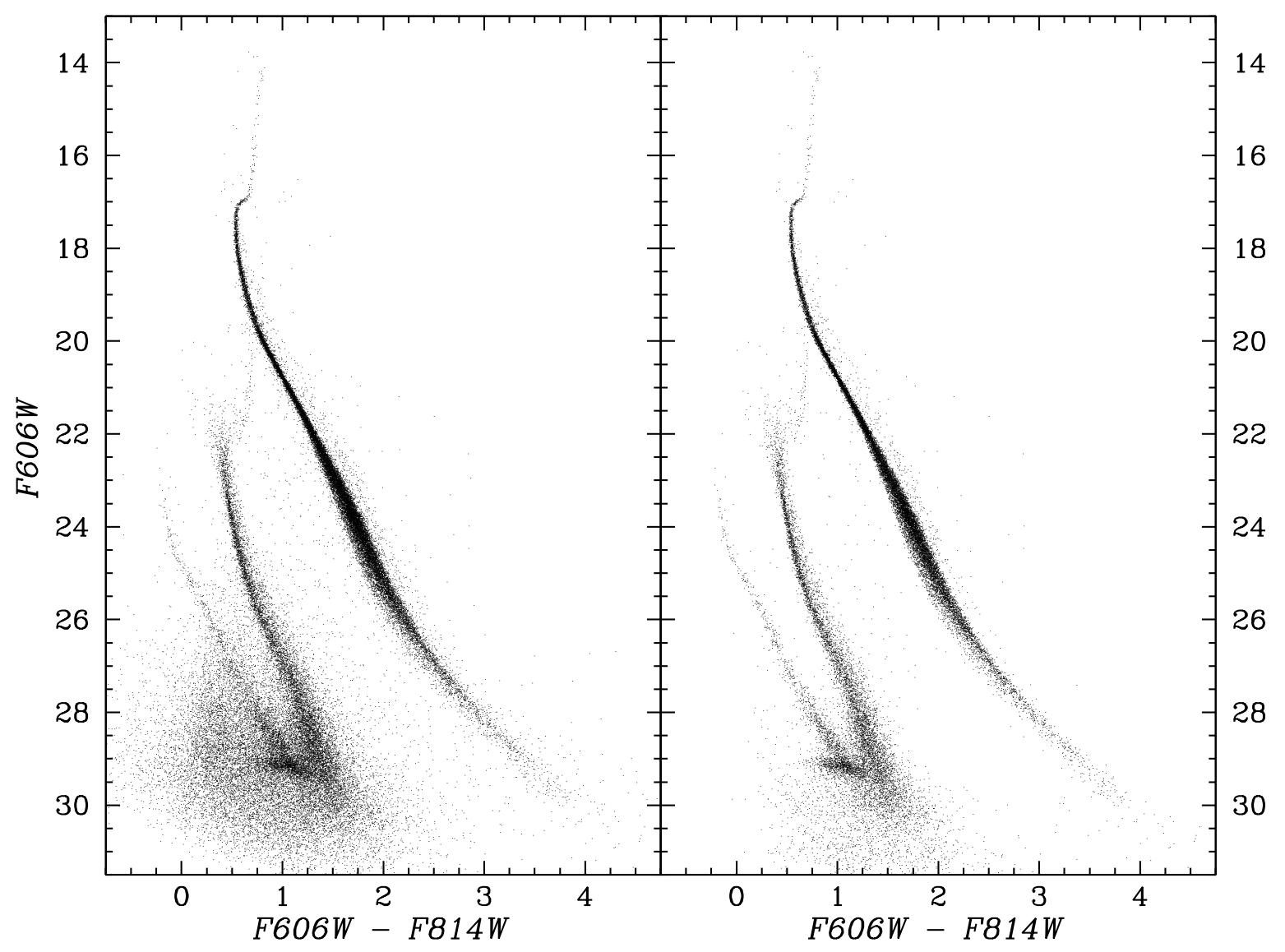

FIG. 10.- The CMD of all stars along the sightline in the ACS primary field, constructed from PSF-fitted photometry of a single deep supersampled drizzled image in each of the $F 606 \mathrm{~W}$ and $F 814 W$ filters (plus corresponding coadded shorter exposures). The left panel illustrates all objects that were detected in both filters and the right panel shows those objects that pass a mild photometric error cut. The CMD reveals a very well defined 47 Tuc main-sequence extending from bright giants at $F 606 W=14$ down to faint, low-mass hydrogen burning dwarfs at $F 606 W=30$, as well as a rich white-dwarf cooling sequence extending over 6 magnitudes in the faint-blue part of the diagram with a sharp hook to the blue at $F 606 W \sim 29$. The deep imaging also penetrates past 47 Tuc to reveal over 10,000 stars in the background SMC dwarf galaxy. The SMC population behind the cluster exhibits a very well defined red-giant branch, multiple main-sequence turnoffs, and a rich main-sequence extending down to $>30$ th magnitude $\left(M \lesssim 0.2 M_{\odot}\right)$.

\subsection{ACS Primary Field Visible Light Color Magnitude Diagram}

The CMD shown in Figure 10 includes all stars detected in both filters of the ACS primary field, and reveals three clear stellar populations. The diagram on the right shows a subset of the data that pass a mild photometric error cut to eliminate poor measurements. The rich sequence on the right of this diagram represents the 47 Tuc red giant branch, turnoff, and main-sequence, extending from $F 606 W=14-30$. This sequence includes 47 Tuc stars ranging from bright giants, through the main-sequence turnoff, down to low mass hydrogen burning dwarfs with $M<0.10 M_{\odot}$. The main sequence exhibits variations in its thickness, an obvious binary sequence except at the faintest magnitudes, and mild evidence for a "trickle" of bluer stars on the lower mainsequence at $F 606 W>26$. The interpretation of these features will follow from a full simulation of the mainsequence using updated stellar evolution models by our team.

The second parallel sequence in this CMD below the 47 Tuc main-sequence represents resolved giant and dwarf stars in the background SMC dwarf galaxy. The photometry of this nearby galaxy extends down to $F 606 W>30$ and the catalog contains over 10,000 stars.
These data will enable studies of the mass function of this galaxy's outer population down to stars with $M \sim 0.15$ $-0.20 M_{\odot}$. The main-sequence turnoff also indicates evidence of multiple turnoffs, confirming an extended star formation history in this remote field. The comparison of the deep mass function of the SMC and the field halo population of the Milky Way will reveal important insights on the formation history of low mass stars with very different metallicities.

The primary science goal of this project is to measure the white-dwarf cooling age of 47 Tuc, and compare it to our recent measurements of M4 and NGC 6397 (Hansen et al. 2004; 2007). Bluer than the SMC sequence, the ACS CMD reveals a rich white-dwarf population of the cluster. Towards the bottom of the cooling sequence near $F 606 W \sim 29$ and $F 606 W-F 814 W \sim 1$, a well defined "hook" in the remnant sequence is seen and is brighter than the faintest dwarfs of the SMC. The morphological diagnostics in the reductions indicate that the bulk of the fainter, scattered population of objects in the CMD below the 47 Tuc white dwarfs and SMC main-sequence are distant galaxies. These can be easily removed when analyzing the foreground populations. Further details and investigation of this CMD and theoretical modeling efforts will be provided in Richer et al. (2012, in prep.) 
and Hansen et al. (2012, in prep.). This work is expected to yield the most accurate age for 47 Tuc to date (e.g., by leveraging both white dwarfs and the main-sequence turnoff), the deepest mass function of the cluster, and new insights on cluster binarity and dynamics.

\subsection{WFC3 Parallel Field Color Magnitude Diagrams}

With the exception of the IR Stare field (59 orbits), all of the observations obtained with the WFC3 instrument in this program had integration times of one orbit in the $F 390 W, F 606 W$, and $F 110 W$ filters (two exposures each), and two orbits in the $F 160 W$ filter (four exposures). Most of the fields targetted relatively uncrowded regions of 47 Tuc, as shown in Figure 2. The observations therefore span a unique region of parameter space, not being limited by crowding yet sampling tens of thousands of cluster and background stars over the $>60$ $\operatorname{arcmin}^{2}$ area in the 13 contiguous fields.

\subsubsection{WFC3/UVIS CMD}

The UVIS CMD of the Swath mosaiced fields is shown in the left panel of Figure 11 and contains $\sim 50,000$ stars. The CMD presents a very clean characterization of the 47 Tuc and SMC main-sequences down to $F 390 W=$ 28.5. Whereas the 47 Tuc main-sequence is extremely sharp at $F 390 W=22-23$, the SMC turnoff exhibits evidence of multiple splittings in this remote field indicative of an extended star formation history. The WFC3/UVIS data also uncover a very rich white-dwarf cooling sequence with over 500 stars, extending almost 7 magnitudes from $F 390 W=21.2$ (three stars) down to 28th magnitude.

This rich population of bright white dwarfs in a coeval stellar population represents a valuable sample for testing physical conditions and processes in degenerate objects. The cooling timescale of young white dwarfs is strongly dependent on the rate at which neutrinos are radiated out of the degenerate core, and two of the primary factors that control this radiation are the density and temperature of the star (Itoh et al. 1996). The observed luminosity functions will test the cooling physics of these stars, and even more so when coupled to independent constraints on the temperature and gravity of the stars from ground based spectroscopy. These outer fields of 47 Tuc are relatively sparse, and the white dwarfs are brighter than those observed with Keck/LRIS in Messier 4 , observations that successfully led to the first direct constraints on the mass distribution of population II white dwarfs (Kalirai et al. 2009c). It is worth stressing that the exposure time of these observations was $\sim 2700$ seconds in each filter, split over one dither position (i.e., two images per pointing).

\subsubsection{WFC3/IR CMD}

A review of the astronomical literature shows that few space-based studies of nearby resolved stellar populations have used the IR bandpasses to test stellar evolution and structure theory for low mass stars. For HST, the previous generation IR imaging camera NICMOS was not heavily used to image populations such as globular clusters on account of its small field of view. For Spitzer, the Infrared Array Camera (IRAC) has both sensitivity over the $3-9.5$ micron wavelength range and a wide field of view, but with much lower throughput and $10 \times$ larger pixels than $H S T$ (resulting in poor performance in crowded regimes). The IR channel of WFC3 therefore fills an important gap in our current observational potential, by providing 1) a large field of view of several square arcmin, 2) excellent sensitivity (Kalirai et al. 2009b), 3) a relatively small pixel size leading to high resolution sampling (0.13 arcsec), and 4) excellent temporal and spatial photometric stability (>99\% - Kalirai et al. 2011). ${ }^{17}$

The right hand panel of Figure 11 presents the WFC3/IR CMD of these same Swath fields (i.e., excluding the deep 59 orbit IR Stare field). The CMD has a unique morphology with a strong kink at $F 110 W$ $=19.2$, about 3 magnitudes below the main-sequence turnoff, and a continuation of the sequence below the kink by another 4 magnitudes where it becomes bluer with increasing faintness. This very sharp feature has been detected in two recent ground-based studies, though not with the clarity and statistical significance of these measurements (Sarajedini, Dotter, \& Kirkpatrick 2009; Bono et al. 2010). The kink feature is caused by collisionally-induced absorption by molecular hydrogen in the atmospheres of cool stars with $T_{\text {eff }} \sim 4500 \mathrm{~K}$ and was anticipated in the low mass stellar models of Baraffe et al. (1997, see their Section 4.2 and Figure 7). This feature is also seen on the UVIS CMD as a change in slope of the lower main-sequence near $F 390 \mathrm{~W}$ $=24$; the effect is clearly much stronger in the IR (note the color baseline) because the molecular absorption bands most dramatically influence near-IR wavelengths (Saumon et al. 1994). Because the kink is largely insensitive to age and metallicity, it provides greater constraints on model fits than the optical CMD (Dotter et al. 2012, in prep.). It grants improved leverage on the age and a critical test of stellar models (see, e.g., Section 5 of Sarajedini, Dotter, \& Kirkpatrick 2009). The morphology and location of the main-sequence below the kink also represents an important calibration to establish the masses of low-mass hydrogen burning stars and brown dwarfs in the Galactic field, where metallicity information is not available. Finally, these IR observations of the complete stellar populations of 47 Tuc are an important data point for red population synthesis models at high metallicity.

Fainter than the kink, the main-sequence of 47 Tuc extends to $F 110 W \gtrsim 23$, below which a second sequence emerges with the opposite slope. This represents the SMC main-sequence and it extends in our data set for $\sim 4$ magnitudes in the IR filters. The red-giant branch of the SMC can be seen extending upwards from the tip of the turnoff, through the 47 Tuc main sequence and up towards the bright-red end of the CMD.

To demonstrate the power of studying globular clusters in the IR bandpasses with WFC3, we select a small number of stars on the lower main sequence of the UVIS CMD in the left panel of Figure 11 (shown as red points with $F 390 W=28.1-28.3$ ), and plot the same stars on the IR CMD in the right panel. These cool stars, have colors of $F 390 W-F 606 W>3$, and are therefore much brighter in the IR bandpasses. The same stars map to the main-sequence at $F 110 W=22.2$, well above the

17 The spatial stability and new on-orbit flat fields are described at http://www.stsci.edu/hst/wfc3/analysis/ir_flats 

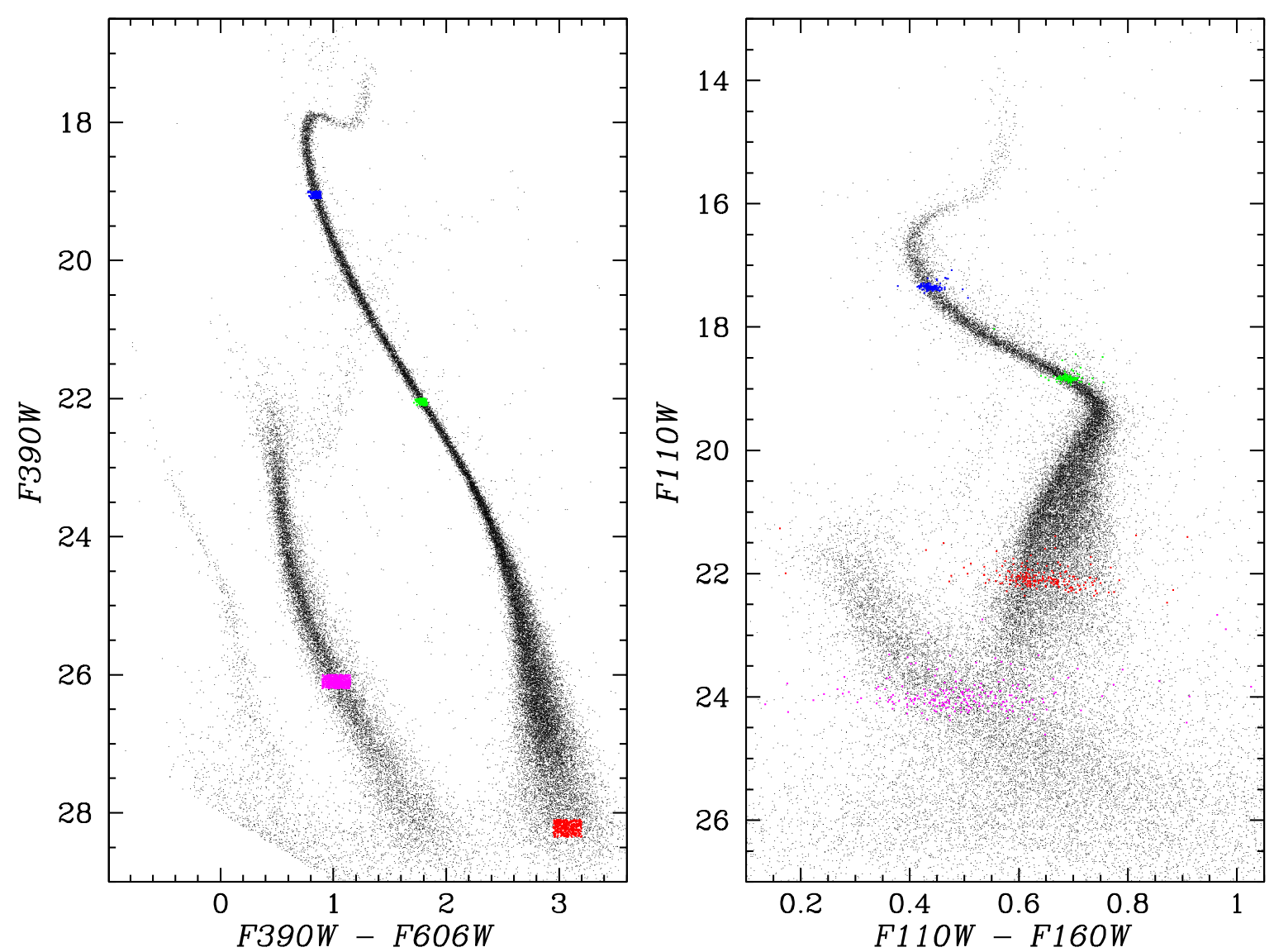

FIG. 11. - CMDs based on the Swath field observations with WFC3/UVIS (left) and WFC3/IR (right) exhibit very distinct morphologies and impressive depths considering the exposure time was a single orbit in each of the $F 390 W, F 606 W$, and $F 110 W$ filters, and two orbits in the $F 160 \mathrm{~W}$ filter (i.e., observations in the 59 orbit IR Stare Field are excluded here). The UVIS CMD clearly highlights the same three populations of stars shown in the ACS primary field (Figure 10); a rich sequence of 47 Tuc red giant branch and main-sequence stars, a background SMC dwarf main-sequence, and a white dwarf cooling sequence stretching over 7 magnitudes. The data extend to beyond 28 th magnitude in the $F 390 W$ filter. The IR CMD, over a narrow color baseline of $<1$ magnitude, shows the rich 47 Tuc main-sequence extending to fainter and redder colors down to $F 110 W=19.2$, beyond which it sharply "kinks" back to the blue and extends another 4 magnitudes to very low masses. Below the 47 Tuc population, the rich main-sequence of the SMC emerges with the opposite slope. To aid in comparing the morphology of the CMDs, the same stars in both panels are color coded along three locations of the 47 Tuc main-sequence and one location of the SMC main-sequence. Further discussion of these CMDs, which combine observations over 13 contiguous fields and contain $\sim 50,000$ stars, is provided in Sections 6.2.1 and 6.2.2

faintest low mass stars that are detected and $\sim 4$ magnitudes above the faintest SMC dwarfs. As most clusters do not have such a rich population of background stars, WFC3/IR imaging with just a few HST orbits can characterize the complete color-magnitude relation of stars and map the main-sequence mass function down to the hydrogen burning limit.

\subsubsection{Panchromatic CMD from $0.4-1.7$ microns}

The final CMD that is presented in Figure 12 combines WFC3/UVIS and IR imaging over all Swath and Stare fields. All objects that were well measured in both the bluest ( $F 390 W$ on WFC3/UVIS) and reddest ( $F 160 W$ on WFC3/IR) filters are selected for this. The full CMD in the top panel extends over a color baseline of $>9$ magnitudes, from $F 390 W-F 606 W=-2$ to 7 . A whitedwarf cooling sequence is mapped over this full baseline, enabling studies of the spectral energy distributions of the remnants, and investigations of objects that show IR excesses from accretion disks or stellar/sub-stellar companions (Woodley et al. 2012, submitted). The 47 Tuc and SMC main-sequences are also very well populated in this CMD, which provides increased leverage to detect multiple splittings or turnoffs. The bottom panel illustrates a closer view of the main-sequence of the cluster stretched over a color baseline of $>4$ magnitudes. Unlike the case of NGC 2808 (e.g., D'Antona et al. 2005; Piotto et al. 2007; see Appendix), this CMD shows no signs of multiple populations for the combined photometric catalog. One caveat to this statement is that, despite the broad wavelength range of this CMD, the $F 390 W$ filter sits at the red end of the UV spectrum and is therefore not sensitive to some elemental variations in the far UV. In addition to the lack of clear splittings in the 47 Tuc CMD, the data indicate no strong spatially dependent variations of the thickness of the cluster sequence across fields separated by large radial distances in the Swath. These initial hints will be fully explored by our team in the near future through a complete study of the stellar and dynamical state of 47 Tuc from these data.

\section{SUMMARY, AND A LOOK FORWARD}

We have presented a detailed analysis of a complex and rich HST data set from Cycle 17 GO program 11677. Over the course of 9 months in 2010, this program spent 121 primary orbits imaging a field with the ACS in- 

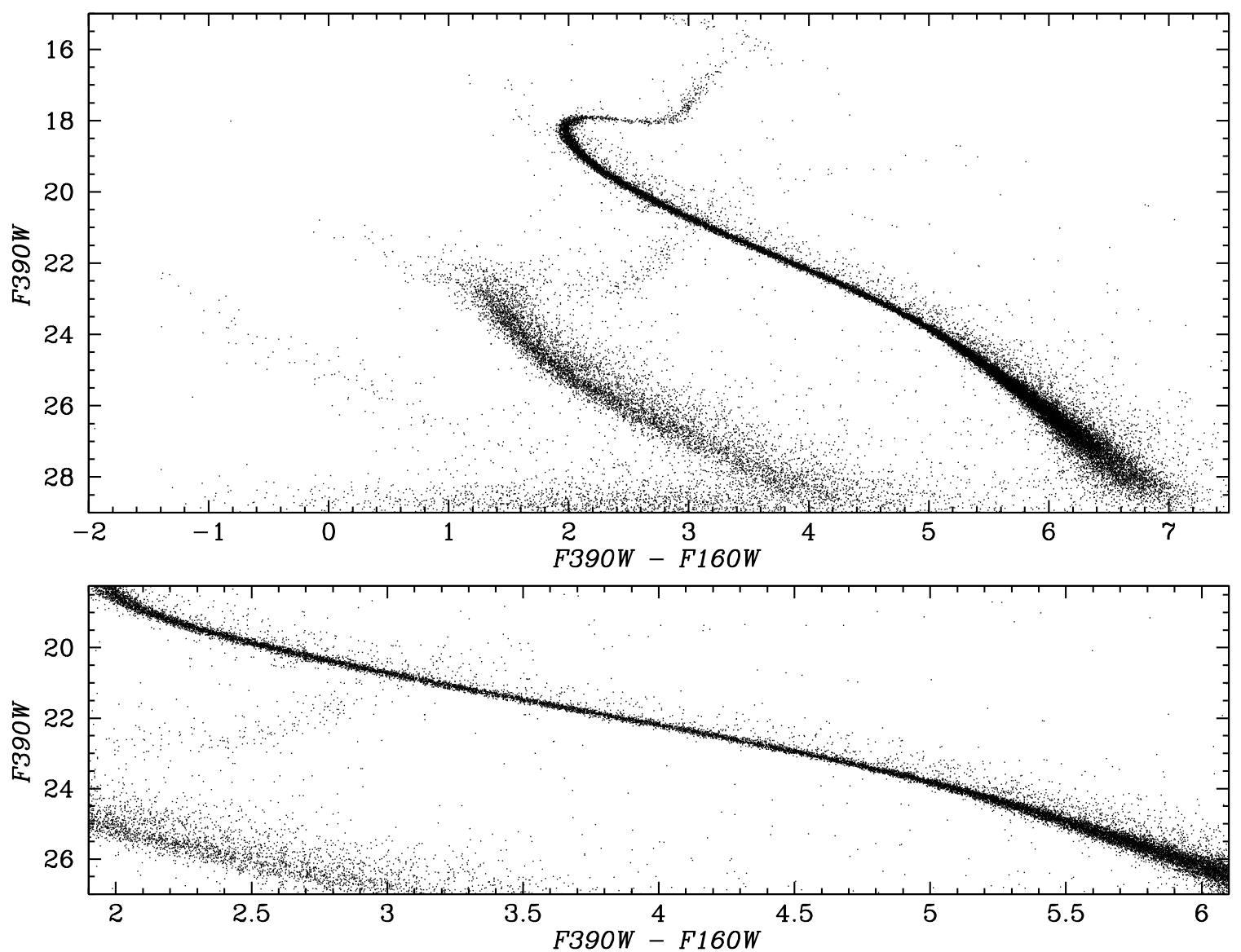

FIG. 12.- The panchromatic nature of this study is highlighted by constructing a CMD of the stellar populations over the widest baseline of $F 390 W-F 160 W$ (i.e., $0.4-1.7$ microns). The combined WFC3/UVIS and IR data stretch the stellar populations over a color range of $>9$ magnitudes (top panel). Despite their faintness in the IR, over 150 white dwarfs form a cooling sequence on this CMD. The bottom panel focuses on the main-sequence of 47 Tuc, which is stretched over $>4$ magnitudes of color.

strument, and 121 parallel orbits imaging multiple fields with both the UVIS and IR cameras of the newly installed WFC3 instrument. These observations were obtained to address science questions that require measuring the complete stellar populations of 47 Tuc, from the faintest hydrogen burning dwarfs, through the mainsequence and giant branches, to the coolest and faintest white dwarf remnants.

We describe the observational design of this program and analyze over 700 full frame HST images using conventional techniques. Our analysis used the MultiDrizzle image analysis package to produce co-added images that are well sampled and free of cosmetic defects. These single images in each filter were then subjected to iterative PSF-fitted photometry using the DAOPHOT and ALLSTAR programs to yield the final photometric, astrometric, and morphological catalogs. The stack-based approach presented in this paper for finding and measuring the faintest stars in the field is fundamentally different from alternative approaches that analyze the raw pixel data in all the images simultaneously. The other analysis approach is underway and will be presented in an upcoming paper (Anderson et al. 2012). The final catalogs and processed images of all fields will be publically released in the near future, upon completion of the science program.

Through our experimentation, we found that three of the most critical steps that affect the quality of the out- put drizzled image was the accuracy of the input image registration, the calculation of the sky offsets between all input images, and the final balance achieved between the pixfrac and scale parameters. Currently, the HST pipeline only produces drizzled images for input exposures obtained within the same visit (typically those that use the same guide stars), without any empirical corrections for incidental image shifts, rotations, or scale changes. For pipeline processing of crowded field images, a more careful consideration of the background variations in the sky level is also needed. Accurately measuring these offsets leads to a smaller standard deviation in the input images and better sigma clipping of deviant pixels. The pipeline reductions also only produce images at a pixfrac of unity and with no rescaling, leading to a gross loss of resolution. Based on our experience, the quality of such images can be greatly improved by using the full capabilities of MultiDrizzle in post-processing, and so several of the key steps are explained in detail in Sections 3.1 and 3.2 (see also "The MultiDrizzle Handbook" - Fruchter \& Sosey 2009). Some of this discussion is especially valuable to WFC3 users, as this program likely represents the most comprehensive test of the image-analysis software on data from the new instrument (e.g., the 59 orbit IR Stare field).

Related to photometric methods, first, we found that simple (small) aperture photometry on the final drizzled images yields an excellent CMD that is not grossly dif- 
ferent from that achieved with careful PSF-fitting with DAOPHOT. Our method does improve such first order photometry by building a PSF from isolated, bright stars on the frame, where the input catalogs were screened several times to remove objects with anomalous measurements. This method has the advantage over techniques where a temporally-varying PSF is derived for each of the individual images in that only a single average PSF is measured on the final stack. A key disadvantage in some implementations of working with drizzled images is that the degree of spatial variation mapped across the field may not be appropriate for $H S T$, for example, if not enough stars are present in the field. In the drizzled images of this program, much of the fine-scale variation of the PSF is averaged out through the combination of many images with individual PSFs at different orientations and detector locations.

The final photometric catalogs contain over 70,000 stars in the ACS primary field and over 50,000 stars in the WFC3 Swath fields. The CMDs from the single drizzled images exhibit tightly defined sequences of the 47 Tuc main-sequence, background SMC main-sequence, and the 47 Tuc white dwarf cooling sequence. The photometry in the primary ACS field extends to >30th magnitude in $F 606 \mathrm{~W}$ and reveals both very low-mass hydrogen-burning stars in the cluster and very cool white dwarf remnants. The parallel WFC3/UVIS data, with exposure times of just 1 orbit per filter per field, exhibit a similar CMD showing the three populations well separated. The sensitivity of the WFC3/UVIS camera is excellent in these relatively crowded regimes, reaching depths of $F 390 W=28.5$ and $F 606 W=29.0$ in a single orbit of integration. ${ }^{18}$

The WFC3/IR observations in this program represent the deepest IR probe of a resolved stellar population to date. Very low mass 47 Tuc stars are measured over $60 \operatorname{arcmin}^{2}$ with just 3 orbits of integration per field (1 orbit in $F 110 W$ and 2 orbits in $F 160 W$ ). The stellar main-sequence is clearly seen to $F 110 W \gtrsim 23$, and the photometry extends several magnitudes below this. The

\footnotetext{
18 The $F 606 W$ photometry shown in Figure 11 includes only those objects that were also detected in the shallower $F 390 \mathrm{~W}$ im-
}

morphology of the CMD shows a distinct "kink" on the lower main sequence, below which the sequence becomes bluer in $F 110 W-F 160 W$ with lower luminosity. The kink is caused by an opacity effect in the atmospheres of cool stars that develop molecular hydrogen at $T_{\text {eff }}=$ $4,300 \mathrm{~K}$. This feature will provide added leverage to a range of studies where CMDs are modeled to constrain stellar evolution and structure theory, as well as cool atmosphere models.

The WFC3/IR CMD of 47 Tuc also provides encouragement for studies of resolved stellar populations with the James Webb Space Telescope (JWST). The NIRCam (and NIRISS) instruments on JWST will be sensitive to optical and IR wavelengths extending from $0.6-5.0 \mathrm{mi}-$ crons. Through both a short and long wavelength channel, NIRCam will also be diffraction limited at both 2 and 4 microns. Relative to HST WFC3/IR, the instrument will have smaller pixels by a factor of 4 , a larger field of view by more than a factor of two, and much higher overall total system throughput. For example, in $\sim 10,000$ seconds of exposure time, the complete stellar populations in dense star clusters down to the hydrogen burning limit will be easily measured out to systems beyond $50 \mathrm{kpc}$ in the Milky Way (e.g., $J=30$ th magnitude).

We wish to thank Anton Koekemoer and Andy Fruchter for numerous discussions related to the use of MultiDrizzle. We wish to thank Stéphane Guisard for providing us with his beautiful wide-field ground based image of the SMC and 47 Tuc. Support for program GO11677 was provided by NASA through a grant from the Space Telescope Science Institute, which is operated by the Association of Universities for Research in Astronomy, Inc., under NASA contract NAS 5-26555. JSK was supported for this work through an STScI Director's Discretionary Fund grant. HBR is supported by grants from The Natural Sciences and Engineering Research Council of Canada and by the University of British Columbia.

\section{APPENDIX}

The ACS data set presented in this paper is unlike most HST investigations of resolved stellar populations. Many more images of 47 Tuc were collected in our program than are typically available, and this provides exquisite screening of deviant pixels and leads to clean, ultra-deep stacks of the data. To test the specific methods described above on more typical data sets, we re-analyzed HST/ACS archive observations of the rich globular cluster NGC 2808 from MAST (GO-10922; PI. G. Piotto). These observations consist of just four dithered exposures in $F 475 W$ and six dithered exposures in $F 814 W$, each with exposure times of 350 - 360 s. A single short observation was also obtained in each filter for the brighter stars.

The results from the analysis of the GO-10922 data are fully described in Piotto et al. (2007). From careful PSFfitted photometry using the methods of Anderson et al. , Piotto et al. are able to resolve NGC 2808's main-sequence into three distinct sequences. Our independent analysis of these data is shown in Figure A1. This includes iterative image registration and sky subtraction, stacking with MultiDrizzle, and PSF-fitted photometry with DAOPHOT and ALLSTAR. All of the steps were done in an automated way, and the total analysis took $<10$ hours from the time the data were retrieved from the archive to the final zero-point calibrated CMD.

The cluster main-sequence on the CMD in Figure A1 extends down to $~ 28$ th magnitude and shows a rich mainsequence with multiple splittings, converging at the turnoff. A white dwarf cooling sequence is also revealed in the faint-blue part of the CMD, previously not reported. The panel on the right shows a zoomed region of the CMD focused on the main sequence, and illustrates the three distinct splittings. Unlike the Piotto et al. analysis, we have not proper motion cleaned these data and have not corrected for differential reddening effects, both of which would make the delineation of the sequences stronger. This simple test confirms that the methods described in this paper, 
when properly implemented, can yield high precision photometry in crowded regimes for data sets with a handful of exposures collected over $1-2$ orbits.
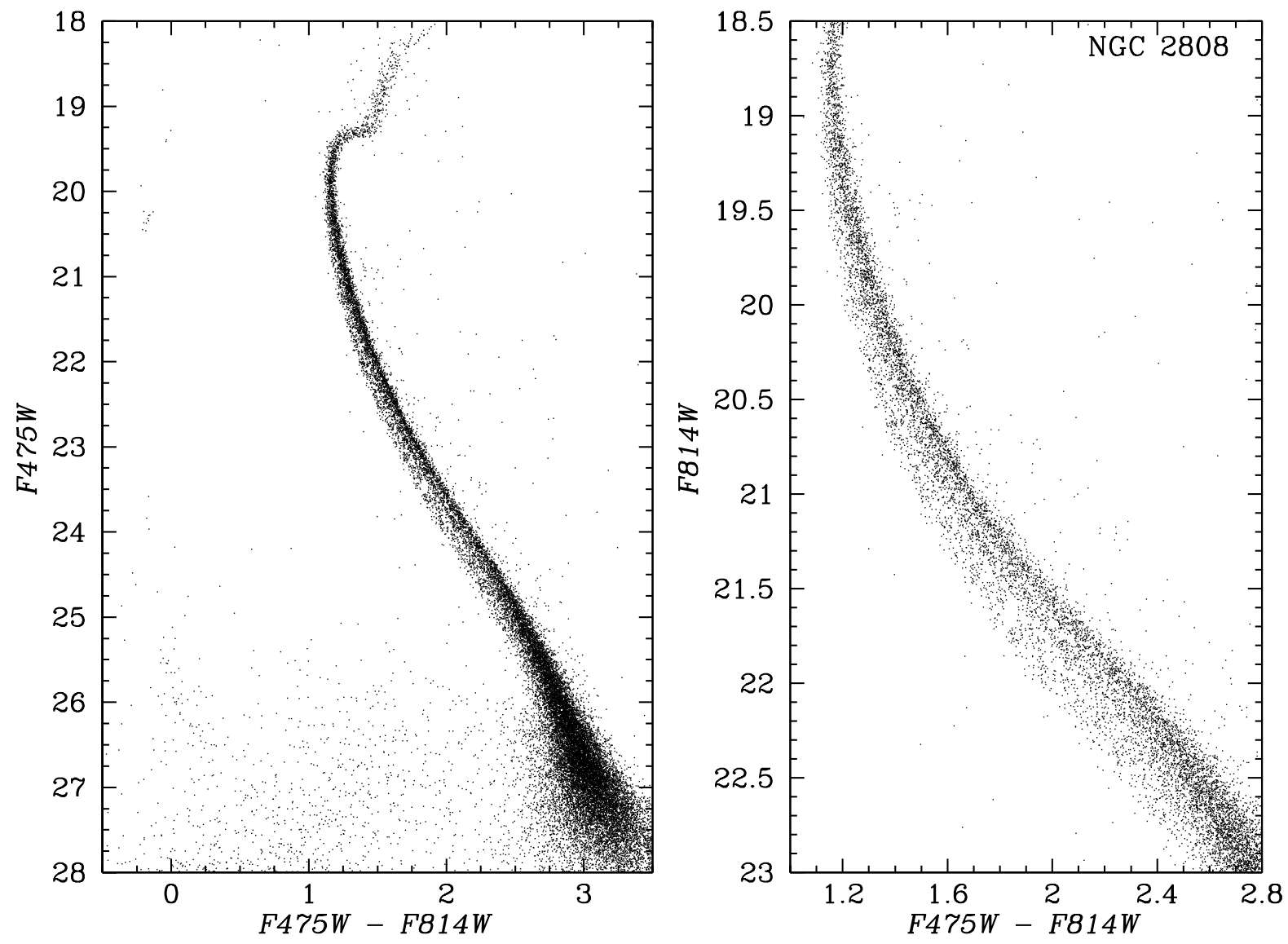

FIG. A1. - The specific methods described in this paper to register images, subtract sky, combine distortion free frames, and perform iterative PSF-fitted photometry are applied to the GO-10922 HST/ACS archive observations of NGC 2808 (see Piotto et al. 2007). These observations are very different from our 47 Tuc ACS data, and consist of just a handful of exposures in each of the two filters, with exposure times of $350-360 \mathrm{~s}$. Through a fully automated analysis, we measure the main-sequence of the cluster down to 28 th magnitude and a white dwarf cooling sequence. A closer look at the cluster's main-sequence shows the three main-sequences reported by Piotto et al. (2007). These data have not been proper motion cleaned and have not been corrected for differential reddening.

\section{REFERENCES}

Anderson, J., \& King, I. R. 2000, PASP, 112, 1360

Anderson, J., et al. 2008a, AJ, 135, 2114

Anderson, J., et al. 2008b, AJ, 135, 2055

Anderson, J., \& Bedin, L. R. 2010, PASP, 122, 1035

Baraffe, I., Chabrier, G., Allard, F., \& Hauschildt, P. H. 1997, A\&A, 327, 1054

Bedin, L. R., Salaris, M., Piotto, G., Anderson, J., King, I. R., \& Cassisi, S. 2009, ApJ, 697, 965

Bohlin, R. 2007, ACS ISR 2007-06, "Photometric Calibration of the ACS CCD Cameras"

Bono, G., et al. 2010, ApJ, 708, L74

Brodie, J. P., \& Strader, J. 2006, ARA\&A, 44, 193

Brown, T M., Ferguson, H. C., Smith, E., Kimble, R. A., Sweigart, A. V., Renzini, A., Rich, R. M., \& VandenBerg, D. A. 2003, ApJ, 592, 17

Bruzual, G. A., \& Charlot, S. 2003, MNRAS, 344, 1000

Carretta, E., Bragaglia, A., Gratton, R., D'Orazi, V., \& Lucatello, S. 2009, A\&A, 508, 695

D'Antona, F., Bellazzini, M., Caloi, V., Fusi Pecci, F., Galleti, S., \& Rood, R. T. 2005, ApJ, 631, 868

Davis, D. S., Richer, H. B., King, I. R., Anderson, J., Coffey, J., Fahlman, G. G., Hurley, J., \& Kalirai, J. S. 2008a, MNRAS, 383, L20

Davis, D. S., Richer, H. B., Anderson, J., Brewer, J., Hurley, J., Kalirai, J. S., Rich, R. M., \& Stetson, P. B. 2008b, AJ, 135, 2155
Demarque, P., \& McClure, R. D. ApJ, 213, 716

Dotter, A., Chaboyer, B., Jevremovic, D., Kostov, V., Baron, E., \& Ferguson, J. W. 2008, ApJS, 178, 89

Dotter, A., et al. 2010, ApJ, 708, 698

Dressel, L., et al. 2010, Wide Field Camera 3 Instrument Handbook, Version 3.0, (Baltimore: STScI)

Fruchter, A. S., \& Hook, R. N. 2002, "A Method for the Linear Reconstruction of Undersampled Images", PASP, 114, 144

Fruchter, A., \& Sosey, M., et al. 2009, "The MultiDrizzle Handbook", version 3.0, (Baltimore, STScI)

Gilliland, R. L., et al. 2000, ApJ, 545, 47

Grogin, N. A., Lim, P. L., Maybhate, A., Hook, R. N., \& Loose, M. 2010, HST Calibration Workshop, "Post-SM4 ACS/WFC Bias Striping: Characterization and Mitigation"

Guhathakurta, P., Yanny, B., Schneider, D. P., \& Bahcall, J. N. 1992, AJ, 104, 1790

Hansen, B. M. S., et al. 2004, ApJS, 155, 551

Hansen, B. M. S., et al. 2007, ApJ, 671, 380

Harris, W. E. 1996, AJ, 112, 1487

Hartig, G. F. 2009a, WFC3 ISR 2009-37 (CAL11437, CAL11439), "WFC3 SMOV Programs 11437/9: IR On Orbit PSF Evaluation"

Hartig, G. F. 2009b, WFC3 ISR 2009-38 (CAL11436, CAL11438), "WFC3 SMOV Programs 11436/8: UVIS On Orbit PSF Evaluation" 
Heinke, C. O., Grindlay, J. E., Edmonds, P. D., Cohn, H. N., Lugger, P. M., Camilo, F., Bogdanov, S., \& Freire, P. C. 2005, ApJ, 625, 796

Hillenbrand, L. A. 1997, AJ, 113, 1733

Holtzman, J. A., Burrows, C. J., Casertano, S., Hester, J. J., Trauger, J. T., Watson, A. M., \& Worthey, G. 1995, PASP, 107, 1065

Hurley, J., et al. 2008, AJ, 135, 2129

Itoh, N., Hayashi, H., Nishikawa, A., \& Kohyama, Y. 1996, ApJS, 102,411

Kalirai, J. S., et al. 2004, ApJ, 601, 277

Kalirai, J. S., et al. 2007, ApJ, 657, L93

Kalirai, J. S., et al. 2009a, WFC3 ISR 2009-31 (CAL11450), "WFC3 SMOV Proposal 11450: The Photometric Performance and Calibration of WFC3/UVIS"

Kalirai, J. S., et al. 2009b, WFC3 ISR 2009-30 (CAL11451), "WFC3 SMOV Proposal 11451: The Photometric Performance and Calibration of WFC3/IR"

Kalirai, J. S., Davis, D. S., Richer, H. B., Bergeron, P., Catelan, M., Hansen, B. M. S., \& Rich, R. M. 2009c, ApJ, 705, 408

Kalirai, J. S., \& Richer, H. B. 2010, Philosophical Transactions of the Royal Astronomical Society, 368, 755

Kalirai, J. S., Cox, C., Dressel, L., Fruchter, A., Hack, W., Kozhurina-Platais, V., \& Mack, J. 2010b, WFC3 ISR-2010-08, "WFC3 Pixel Area Maps"

Kalirai, J. S., Deustua, S., Rajan, A., \& Riess, A. 2011, WFC3 ISR 2011-08, "The Photometric Performance of WFC3/IR: Temporal Stability Through Year 1"

King, I. R., Anderson, J., Cool, A. M., Piotto, G. 1998, ApJ, 492, L37

Koekemoer, A. M., et al. 2002, "HST Dither Handbook", Version 2.0, Baltimore: STScI

Lauer, T. R. 1999a, PASP, 111, 1434
Lauer, T. R. 1999b, PASP, 111, 227

Maiz Apellaniz, J. 2007, ASP Conference Series, 364, 227

Marín-Franch, A. et al. 2009, ApJ, 694, 1498

Massey, R., Stoughton, C., Leauthaud, A., Rhodes, J., Koekemoer, A., Ellis, R., \& Shaghoulian, E. 2010, MNRAS, 401, 371

McLaughlin, D. E., Anderson, J., Meylan, G., Gebhardt, K., Pryor, C., Minniti, D., \& Phinney, S. 2006, ApJS, 166, 249

Meylan, G., \& Mayor, M. 1986, A\&A, 166, 122

Piotto, G., Bedin, L. R., Anderson, J., King, I. R., Cassisi, S., Milone, A. P., Villanova, S., Pietrinferni, A., \& Renzini, A. 2007, ApJ, 661, L53

Piotto, G. 2009, IAU Symposium 258, 233

Rajan, A., et al. 2010, "WFC3 Data Handbook", Version 2.0, (Baltimore: STScI)

Renzini, A., \& Fusi Pecci, F. 1988, ARA\&A, 26, 199

Richer, H. B. et al. 2004, AJ, 127, 2771

Richer, H. B. et al. 2008, AJ, 135, 2141

Sandage, A. R. 1953, AJ, 58, 61

Sarajedini, A., et al. 2007, AJ, 133, 1658

Sarajedini, A., Dotter, A., \& Kirkpatrick, A. 2009, ApJ, 698, 1872

Saumon, D., Bergeron, P., Lunine, J. I., Hubbard, W. B., \& Burrows, A. 1994, ApJ, 424, 333

Shapley, H. 1917, ApJ, 45, 118

Sirianni, M., et al. 2005, PASP, 117, 1049

Stetson, P. B. 1987, PASP, 99, 191

Stetson, P. B. 1992, ASP Conference Series, 25, 297

Stetson, P. B. 1994, PASP, 106, 250

VandenBerg, D. A., \& Bell, R. A. 1985, ApJS, 58, 561

Whitmore, B. C., \& Schweizer, F. 1995, AJ, 109, 960

Zoccali, M. et al. 2001, ApJ, 553, 733 